Engineering Physics and Mathematics Division

Mathematical Sciences Section

\title{
A NEW SHARED-MEMORY PROGRAMMING PARADIGM FOR MOLECULAR DYNAMICS SIMULATIONS ON THE INTEL PARAGON
}

\author{
E.F. D'Azevedo \\ C.H. Romine \\ Mathematical Sciences Section \\ Oak Ridge National Laboratory \\ P.O. Box 2008, Bldg. 6012 \\ Oak Ridge, TN 37831-6367
}

Date Published: December 1994

Research supported by the Applied Mathematical Sciences subprogram of the Office of Energy Research, U.S. Department of Energy

Prepared by the Oak Ridge National Laboratory Oak Ridge, Tennessee 37831 managed by Martin Marietta Energy Systems, Inc. for the U.S. DEPARTMENT OF ENERGY under Contract No. DE-AC05-84OR21400 



\section{DISCLAIMER}

This report was prepared as an account of work sponsored by an agency of the United States Government. Neither the United States Government nor any agency thereof, nor any of their employees, make any warranty, express or implied, or assumes any legal liability or responsibility for the accuracy, completeness, or usefulness of any information, apparatus, product, or process disclosed, or represents that its use would not infringe privately owned rights. Reference herein to any specific commercial product, process, or service by trade name, trademark, manufacturer, or otherwise does not necessarily constitute or imply its endorsement, recommendation, or favoring by the United States Government or any agency thereof. The views and opinions of authors expressed herein do not necessarily state or reflect those of the United States Government or any agency thereof. 


\section{DISCLAIMER}

Portions of this document may be illegible in electronic image products. Images are produced from the best available original document. 


\section{Contents}

1 Introduction . . . . . . . . . . . . . . 1

1.1 DOLIB (Distributed Object Library) $\ldots \ldots \ldots \ldots \ldots \ldots$

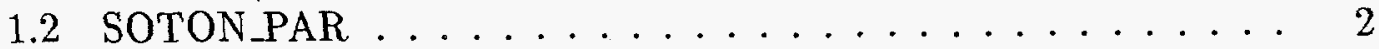

2 Overview of Parallelization of SOTON_PAR with DOLIB . . . . . . 3

2.1 Geometric Hashing . . . . . . . . . . . . . . 3

2.2 DOLIB implementation of movout . . . . . . . . . . 4

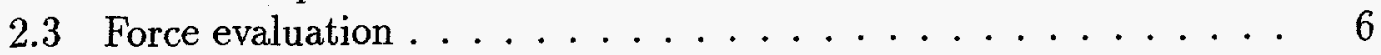

2.4 Dynamic Load Balancing . . . . . . . . . . . . . . . . 7

2.5 Shifted Force Potential . . . . . . . . . . . . . . 8

3 Parallel Performance . . . . . . . . . . . . . . . 8

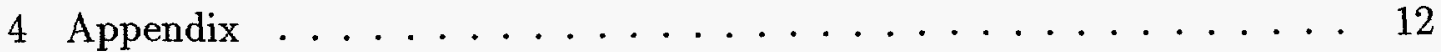

4.1 subroutine movout $\ldots \ldots \ldots \ldots \ldots \ldots \ldots \ldots \ldots \ldots$

4.2 subroutine adjustxyz $\ldots \ldots \ldots \ldots \ldots \ldots \ldots \ldots$

4.3 subroutine hashxyz . . . . . . . . . . . . 15

4.4 subroutine setxlist . . . . . . . . . . . . . 19

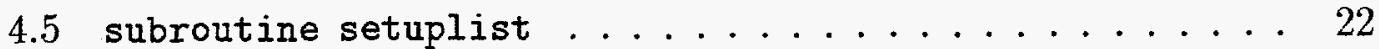

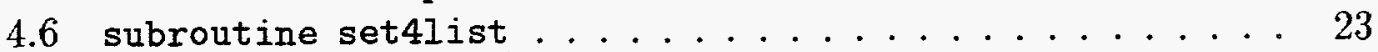

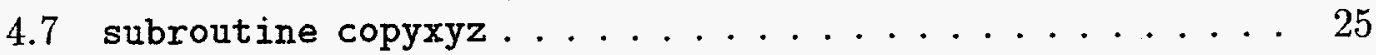

4.8 subroutine force . . . . . . . . . . . . . 29

4.9 subroutine forceall . . . . . . . . . . . . 31

4.10 subroutine forcecol . . . . . . . . . . . . 33

4.11 subroutine forcecal . . . . . . . . . . . . 45

5 References ........................ 51 



\section{List of Figures}

2.1 Top view of columns used in processing column $(i, j)$. Only two new columns are needed in processing column $(i+1, j) \ldots \ldots 7$ 



\title{
A NEW SHARED-MEMORY PROGRAMMING PARADIGM FOR MOLECULAR DYNAMICS SIMULATIONS ON THE INTEL PARAGON
}

\author{
E.F. D'Azevedo \\ C.H. Romine
}

\begin{abstract}
This report describes the use of shared memory emulation with DOLIB (Distributed Object Library) to simplify parallel programming on the Intel Paragon. A molecular dynamics application is used as an example to illustrate the use of the DOLIB shared memory library. SOTON_PAR, a parallel molecular dynamics code with explicit message-passing using a Lennard-Jones 6-12 potential, is rewritten using DOLIB primitives. The resulting code has no explicit message primitives and resembles a serial code. The new code can perform dynamic load balancing and achieves better performance than the original parallel code with explicit messagepassing.
\end{abstract}




\section{Introduction}

This report describes the use of shared memory emulation provided by DOLIB (Distributed Object Library) [3] for parallel programming of large-scale Molecular Dynamics (MD) codes on the Intel Paragon distributed memory supercomputer. SOTON_PAR [7], a parallel MD code that uses explicit message passing and Lennard-Jones atoms (6-12 potential), is rewritten using DOLIB shared memory primitives. The new MD prototype code can perform dynamic load balancing and achieves better performance than the original code that uses spatial decomposition. This report may also serve as a guide for scientists or programmers considering the use of DOLIB shared memory emulation in more sophisticated molecular dynamics simulations or particle-in-cell methods.

While this prototype code uses a simple Lennard-Jones 6-12 potential, more complicated force evaluation (such as including angular (three-body) forces for silicon and torsional (four-body) forces for organic polymers or proteins) can easily be added using the DOLIB shared memory framework. DOLIB's dynamic load balancing capability is especially attractive for more demanding MD simulations.

\subsection{DOLIB (Distributed Object Library)}

DOLIB (Distributed Object Library) is a set of FORTRAN and C callable routines to emulate shared memory on distributed-memory environments such as Intel multiprocessors and PVM clusters of workstations.

DOLIB supports runtime dynamic creation and destruction of one-dimensional arrays. Explicit gather and scatter operations provide access to array elements. DOLIB is portable since no language extension is introduced and no preprocessor, compiler or operating system support is required. DOLIB provides an atomic accumulate operation axpby $(y($ index $(:) \leftarrow \alpha * x(:)+\beta * y($ index $(:))$, where $x$ is a local array, $y$ is a globally shared array, and index is an array of indices for $y$. axpby is intended for use in finite element matrix assembly, with no need for explicit lock/unlock. Note that $\alpha=1, \beta=0$ corresponds to a scatter operation. DOLIB also provides automatic caching of read-only data to reduce 
message traffic. However, the programmer has the responsibility of flushing the cache to maintain coherency during concurrent updates.

A global array in DOLIB is stored as fixed-size pages in a block wrapped mapped fashion across all processors. DOLIB translates requests for remote data (gather) or update (scatter) into the appropriate message sent to the "owner" processor of that data page. These DOLIB requests are then serviced by the IPX [6] message system.

DOLIB supports a generalized atomic update operation (axpbyz),

$$
\begin{aligned}
z(:) & \leftarrow y(\text { index }(:)) \\
y(\operatorname{index}(:)) & \leftarrow \alpha * x(:)+\beta * y(\text { index }(:)) \\
x(:) & \leftarrow z(:)
\end{aligned}
$$

Operation axpbyz can also be used to implement a vector of counting semaphores.

\subsection{SOTON_PAR}

The original version of SOTON_PAR ${ }^{1} \mathrm{MD}$ code ran on the Intel iPsc/2. The version we obtained was substantially modified by David Walker to run efficiently on Intel Paragon multiprocessors. SOTON_PAR models short range inter-atomic interactions by using a link-cell (geometric hashing) algorithm where all particles are hashed into three-dimensional $N_{b} \times N_{b} \times N_{b}$ bins. The bin size is at least the cut-off distance $\left(r_{c}\right)$ used in the short-range force evaluation. SOTON_PAR exploits the symmetry of Newton's Third Law by examining atoms in only 13 (instead of 26) neighboring cells. It uses a spatial decomposition [8] and requires message exchanges with neighbor processors at each time step as atoms migrate across processor boundaries. Many of the MD algorithms used in SOTON_PAR are described in the book Computer Simulation of Liquids [1]. The code also uses a "shifted-force" [9] Lennard-Jones 6-12 potential to avoid a discontinuity at the

\footnotetext{
${ }^{1}$ The source code for SOTON_PAR is available from the CCP5 archive at ftp://ftp.dl.ac.uk/ecp5/SOTON.PAR/mdplli3cu.master
} 
cut-off distance.

The code consists of two parts: the "host" code runs on the service partition and controls the I/O, allocation and loading of nodes etc.; the "worker" code runs on the compute nodes and performs most of the work in geometric hashing and force evaluations.

The code assumes an $N_{c} \times N_{c} \times N_{c}$ FCC (Face Centered Cubic) lattice scaled to the canonical unit cube $[-0.5,0.5] \times[-0.5,0.5] \times[-0.5,0.5]$, with a total of $N=4 N_{\mathrm{c}}^{3}$ atoms. A periodic boundary condition is imposed on all sides. A simple Verlet leap-frog time stepping scheme is used to update the particle positions.

\section{Overview of Parallelization of SOTON_PAR with DOLIB}

We have written a new parallel MD prototype code based on SOTON_PAR using DOLIB shared memory primitives. The new code shares much of the overall structure of SOTON_PAR with most of the host code for performing I/O executed by node 0 . We believe the new code is much easier to write and understand without the complexities of explicit message passing code. We have also added several enhancements: (1) initialize with Boltzmann distribution for faster convergence to equilibrium, (2) a more robust parallel cold-start mechanism even for very large configurations, (3) an option for dynamic load balancing, (4) an option for using double precision for force computation.

We shall discuss two of the most time consuming computational kernels in SOTON_PAR: movout, which performs geometric hashing, and force, which performs the force evaluations.

\subsection{Geometric Hashing}

The link-cell method [4] is commonly used to speed up MD force calculations by minimizing the number of neighboring atoms that must be checked for possible interaction (based on a cut-off distance of $r_{c}$, beyond which particle interaction is assumed to be negligible). At each time step, all the atoms are hashed into 
three-dimensional bins or cells of side length at least $r_{c}$. Each particle can thus interact only with atoms within the same bin or the 26 surrounding bins. Hashing the atoms can be easily parallelized and requires only $O(N)$ work. Only 13 of the neighboring bins need to be examined if Newton's Third Law $\left(f_{i j}=-f_{j i}\right)$ is applied. The link-cell method is memory efficient, requiring only $O\left(N_{b}^{3}\right)$ storage for the 3-D bins, 9 real vectors of length $N\left(x, y, z, v_{x}, v_{y}, v_{z}, f_{x}, f_{y}, f_{z}\right.$ for the positions, velocities and forces, respectively) and an integer vector of length $N$ for storing the linked list.

Another common technique is to construct and maintain for each atom, a list of neighboring atoms [10]. This list is updated every few time steps. The advantage provided by the neighbor list is that once the list is built, examining it for possible interaction is much quicker than checking all other atoms in the neighboring bins. However, the neighbor list for each atom commonly grows quite long (70 or more atoms per list), and the resulting high cost in memory is prohibitive for very large scale MD calculations.

SOTON_PAR is based on a spatial decomposition and uses the link-cell method. Each processor is responsible for a spatial region and the corresponding subset of 3-D bins. SOTON_PAR uses message passing code to exchange atoms with neighbor processors as atoms migrate across processor boundaries. A highly nonuniform distribution of particles may result in a serious load-imbalance.

\subsection{DOLIB implementation of movout}

The link-cell algorithm in SOTON_PAR constructs a linked list of atoms for each bin. We implement the link-cell method by performing a reordering or renumbering so that all atoms in a bin are contiguously numbered, e.g., if each bin has 10 atoms, then after the reordering, atoms 1 to 10 are contained in the first bin, atoms 11 to 20 are contained in the second bin, etc. The reordering permits the use of the most efficient DOLIB contiguous block gather/scatter operations on long vectors.

Two passes are required for the geometric hashing and reordering. The first 
pass performs the geometric hashing and stores the result of the mapping. For each bin, we compute the number of atoms to be assigned. We then set up and allocate storage for each bin using a pointer array $x l i s t$. The second pass then performs the actual reordering and data movement. Note that vectors $\left(f_{x}, f_{y}, f_{z}\right)$ are used as temporary storage and will be cleared again for force computation.

The geometric hashing and reordering is performed in several routines:

adjustxyz: After a time step, particles close to the boundary might exit the canonical box $[-0.5,0.5] \times[-0.5,0.5] \times[-0.5,0.5]$. We implement a periodic boundary condition by adjusting the $(x, y, z)$ coordinate to reintroduce the particle at the opposite face. Hence,

$$
\begin{aligned}
& \text { if } \quad(x>0.5) \quad x=x-1.0 \\
& \text { if } \quad(x<-0.5) \quad x=x+1.0
\end{aligned}
$$

hashxyz: Geometric hashing is performed as a simple divide and integer truncation operation. An atom with coordinates $(x, y, z)$ will be hashed to 3-D bin $(i, j, k)$ by

$$
\begin{aligned}
i & =1+\operatorname{int}\left((x-(-0.5)) / r_{c}\right) \\
j & =1+\operatorname{int}\left((y-(-0.5)) / r_{c}\right) \\
k & =1+\operatorname{int}\left((z-(-0.5)) / r_{c}\right)
\end{aligned}
$$

The result of the hashing is stored in array imap for use in the second pass. The array imap requires $N_{b}^{3}$ words of memory.

setxlist: We use a pointer array $x l$ ist to mark for each bin, the beginning position (or label) of each contiguous block of atoms assigned to the bin.

Let $m_{i}$ be the number of particles assigned to the $i^{\text {th }}$ bin, then $x \operatorname{list}(1)=1$, $x \operatorname{list}(2)=1+m_{1}, x \operatorname{list}(3)=x \operatorname{list}(2)+m_{2}, \cdots, x \operatorname{list}(k+1)=x \operatorname{list}(k)+m_{k}$, etc. The array xlist requires $N_{b}^{3}$ words of memory. 
setuplist: We shall use $\mathrm{xl}$ ist as pointer to the next available storage position for each bin. Thus as we assign particles into bins, $x l$ ist has to be incremented in parallel with an atomic operation. We avoid the use of inefficient lock/unlock by using the DOLIB axpbyz operation.

copyxyz: Once the assignment mapping is determined, vectors $(x, y, z)$ and $\left(v_{x}, v_{y}, v_{z}\right)$ are permuted using $\left(f_{x}, f_{y}, f_{z}\right)$ as temporary storage. Vectors $\left(f_{x}, f_{y}, f_{z}\right)$ are then cleared again before the force computation. The arrays $(x, y, z),\left(v_{x}, v_{y}, v_{z}\right)$ and $\left(f_{x}, f_{y}, f_{z}\right)$ require $9 N$ words of memory for $N$ atoms. If the forces $\left(f_{x}, f_{y}, f_{z}\right)$ are to be computed in double precision, $12 N$ words are required.

adjustxlist: Finally we reset $x l$ ist to point to the beginning position of each contiguous block.

Note that as the MD system converges to an equilibrium state, most of the particles will be hashed into the same bins to which they belonged in the previous time step, thus requiring only a small amount of data movement.

In Section 3 we list the proportion of time spent in each routine.

\subsection{Force evaluation}

We take advantage of Newton's 3rd Law $\left(f_{i j}=-f_{j i}\right)$ to compute a force interaction only once for each atom pair. This means only 13 (instead of 26) neighboring bins must be examined. Lomdahl [5] describes the selection of 13 neighbors as a particular "3D interactions path", though in fact any stencil of 13 neighboring bins can be chosen, if consistently used.

For simplicity, we consider a two-dimensional partition of the computation. Thus, we group all force interactions for each column of $N_{b}$ cells of the $3 \mathrm{D}$ mesh of bins as one unit. The $N_{b} \times N_{b}$ columns are then block partitioned and assigned to individual processors. Because all atoms in each bin are contiguously ordered (see Section 2.2), DOLIB's efficient contiguous block gather/scatters can be used. Moreover, we exploit data reuse by selecting the 13 neighboring cells to be 

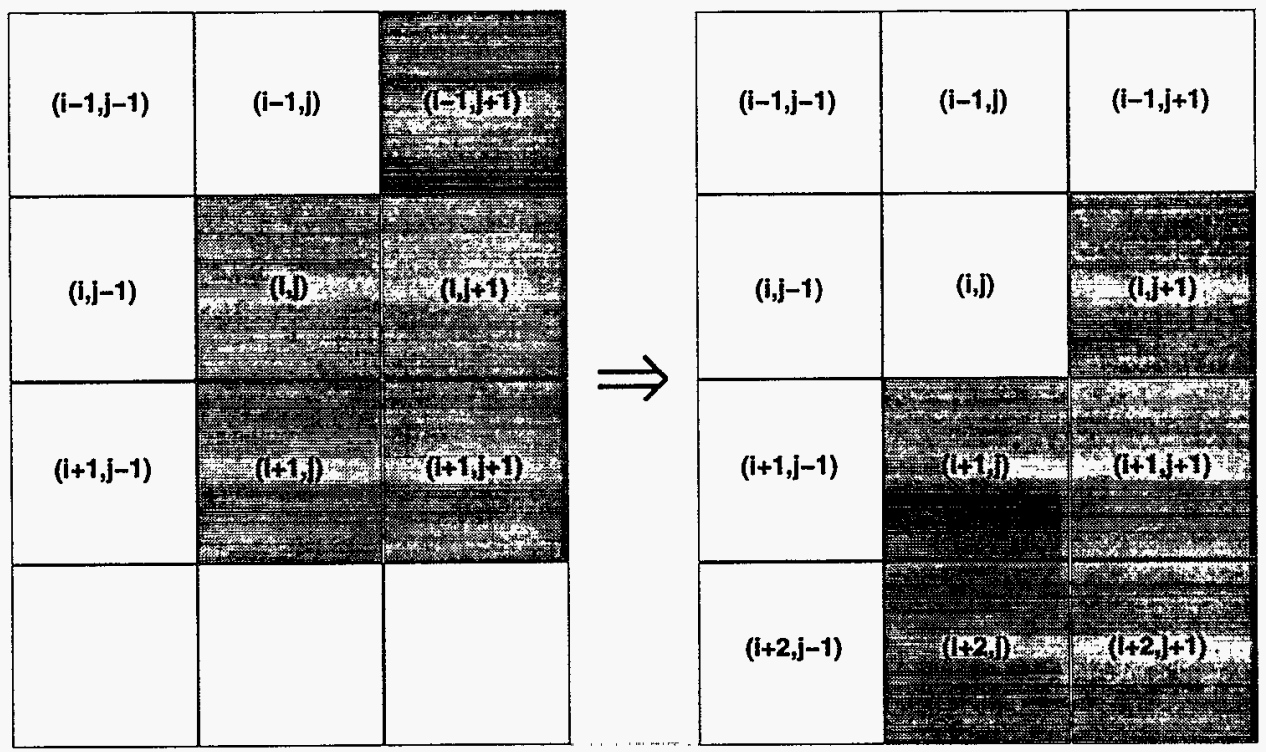

Figure 2.1: Top view of columns used in processing column $(i, j)$. Only two new columns are needed in processing column $(i+1, j)$.

in 5 neighboring columns (see Figure 2.1). To process column $(i, j)$ we require data from neighboring columns $(i-1, j+1),(i, j+1),(i+1, j),(i+1, j+1)$; to process the next column $(i+1, j)$, data in columns $(i+1, j),(i, j+1),(i+1, j+1)$ can be reused. Only data for two new columns $(i+2, j)$ and $(i+2, j+1)$ must be brought in. This organization reduces the amount of communication required for gather/scatter operations.

Another advantage of this reorganization is that force evaluation in each of the bins requires no further communication. Automatic thread parallelization by the Intel MP node Paragon compiler is thus simplified.

\subsection{Dynamic Load Balancing}

We include an option to estimate a work measure for each column, using this to distribute columns to processors to avoid load imbalance. One simple measure of work load is the total number of atoms in the column. For a uniform distribution of atoms in the domain, this is generally sufficient to attain good load-balance. However, for nonuniform distributions, a more reasonable work measure for each 
bin is to count the total number of possible atom-atom interactions among other particles in the 13 neighboring bins. Both of these work estimates are provided as options to the load-balancing routine.

The load balancing strategy then computes the overall total and average amount of work for each processor, assigning columns to processors to satisfy this average using a greedy algorithm. Future versions will provide a more so-

phisticated bin-packing algorithm. However, for the simple Lennard-Jones fluid simulations, each bin has approximately the same number of atoms and induces almost no load imbalance.

\subsection{Shifted Force Potential}

SOTON_PAR implements a shifted force Lennard-Jones 6-12 potential [1, page 145] where a small linear term is added to the potential so that it is continuous and its derivative is zero at the cutoff distance. The code also computes the virial and potential energy along with force calculations. Each atom-pair takes 8 flops and 1 compare to determine whether they are within the cut-off distance. If they are, then another 29 flops, 2 divides and 1 square root operation are further required. In our experience, the accumulation into total potential energy may encounter catastrophic cancellation that can yield as few as 4 significant digits of precision. We have implemented an option using separate double precision variables $v_{p o s}$ and $v_{n e g}$ for accumulating positive and negative potential values, which reduces the amount of numerical cancellation and produces more consistent results as the number of processors is varied.

\section{Parallel Performance}

Our parallel codes were tested on an Intel MP node Paragon system. Each MP node contains 3 CPUs and at least 64MBytes of memory in a local shared memory configuration. By default, one CPU is a dedicated message co-processor. The second CPU runs the main computational thread. The third CPU can be utilized by 
automatic thread parallelization by the Paragon MP Fortran compiler. The MP node can also be configured in "turbo" mode where all three CPUs are dedicated for computation but with some degradation in communication performance. All our MD computations were done in single precision in non-turbo mode, although we have also implemented a double precision option for force computations.

We tested our code with a benchmark problem described in Plimpton [8, page 23] for a Lennard-Jones 6-12 potential with reduced density $\rho=0.8442$, and reduced temperature $T=0.72$. The system is initialized with a fcc lattice and randomized velocities chosen from a Boltzmann distribution. The integration time step is 0.00462 in reduced units, and cut-off distance is $r_{c}=2.5 \sigma$. Plimpton estimates there are about 55 neighbors interacting with each atom at every time step (out of about 177 atom-pairs examined). If we count a divide as 5 flops and a square root also as 5 flops, then (from Section 2.5) each atom requires approximately $9 * 177+44 * 55=4013$ flops for force evaluation. Updating the velocities, positions and accumulation of total kinetic energy in movea and moveb requires another 30 flops per atom.

Table 3.1 shows averaged run times per time step for a 500,000 atom simulation $(50 \times 50 \times 50$ lattice $)$. Initialization and setup time are excluded. We achieved faster run times without the overhead of computing work measure and dynamic load balancing, since the problem is already well-balanced. The serial run was performed using the same "parallel" code on a single processor. Note that no messages were generated in the DOLIB gather/scatter since on a single processor these are translated into memory copies.

For the 500,000 atom problem, our code achieved a speed of $0.175 \mathrm{~ms} /$ atom per time step on a single processor (with parallel threads) with no messages generated. About $75 \%$ of the overall time (per time step) was spent in force computation and about $20 \%$ in performing reordering in movout. Within movout, about $42 \%$ of time was spent in performing 3-D bin hashing (first pass) in hashxyz, about $17 \%$ in allocating storage with DOLIB axpbyz operation in routine setuplist and about $35 \%$ in performing (second pass) actual reordering of vectors $(x, y, z$, 
Table 3.1: Averaged runtimes (in sec) per timestep for 500,000 nodes problem.

\begin{tabular}{|r|r|r|}
\hline Processor & 1 thread & 2 threads \\
\hline \hline 1 & 137.5 & 95.4 \\
2 & 73.1 & 51.8 \\
4 & 37.6 & 27.2 \\
8 & 19.7 & 13.2 \\
16 & 10.3 & 7.1 \\
32 & 5.6 & 4.3 \\
64 & 3.3 & 2.4 \\
\hline
\end{tabular}

$\left.v_{x}, v_{y}, v_{z}\right)$ in routine copyxyz. On 64 processors with parallel threads, the speed decreased to about $0.31 \mathrm{~ms} /$ atom per processor per time step. About $60 \%$ was in force and $30 \%$ in movout. Within movout, about $27 \%$ was in hashxyz, $21 \%$ in setuplist and about $38 \%$ was in copyxyz. Note however, there is message passing and servicing of remote memory requests even within the force computations.

By comparison, the original SOTON_PAR host/node codes required about $17.1 \mathrm{sec}$ on 8 processors for a 256,000 atom $(40 \times 40 \times 40$ lattice $)$ with no parallel threads. This is about $0.534 \mathrm{~ms} /$ atom/processor. Our code (with no parallel threads) takes about $10.4 \mathrm{sec}$ per time step. This is about $0.333 \mathrm{~ms} /$ atom. With parallel threads enabled, the average time reduced to $7.7 \mathrm{sec}$ per step, or $0.246 \mathrm{~ms} /$ atom/processor.

On a much larger problem with 32 million atoms $(200 \times 200 \times 200$ lattice $)$ our code takes about $205.3 \mathrm{sec}$ on 32 processors and $103.7 \mathrm{sec}$ on 64 processors, which is about $0.207 \mathrm{~ms} /$ atom per processor. This yields an approximate overall rate (including message passing overhead) of 19.5MFlop/sec for each node (with parallel threading enabled). Although we used single precision in our codes, our run times compare quite favorably with the times (performed in double precision) of $2.03 \mathrm{~ms} /$ atom/processor on the Intel Delta [8] and $1 \mathrm{~ms} /$ atom/processor [2] on a 1024 processor CM-5 both using a spatial decomposition and linked-cell method. 


\section{Acknowledgments}

The authors take this opportunity to express appreciation to Bob Marr, Ron

Peierls and Joe Pasciak for the IPX package, which simplified the development of DOLIB. We also thank Bill Shelton and David Walker for their insight and advice on Molecular Dynamics Simulations. 


\section{Appendix}

In this appendix, we list the RATFOR source code for some of the key routines used in movout permutation and force computations.

\section{1. subroutine movout}

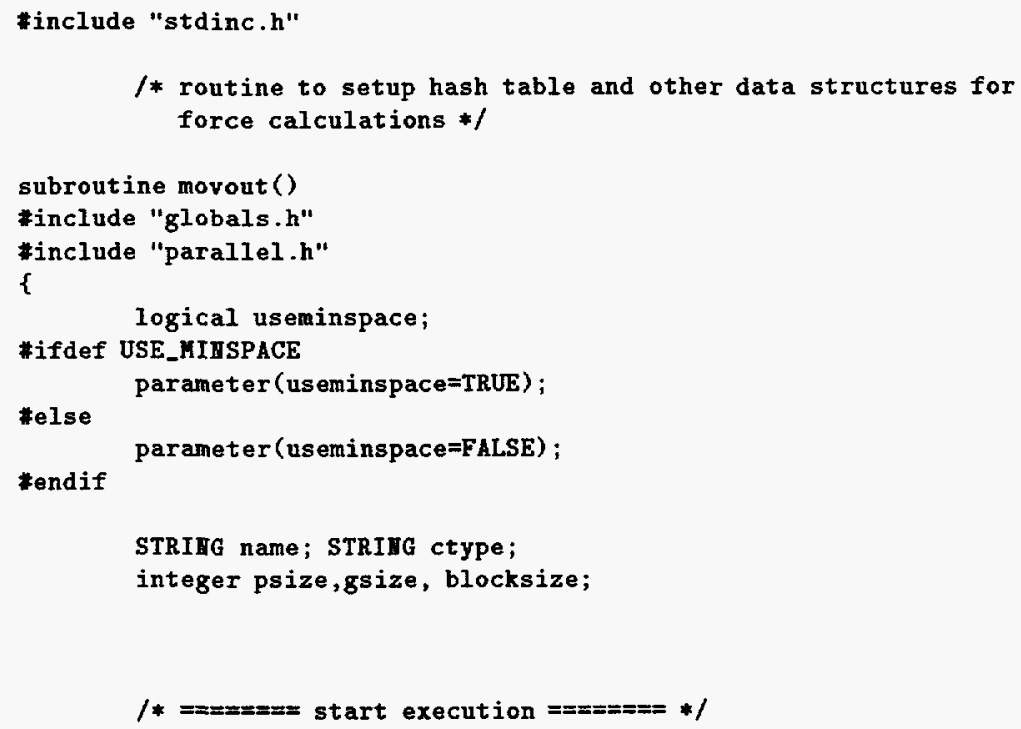




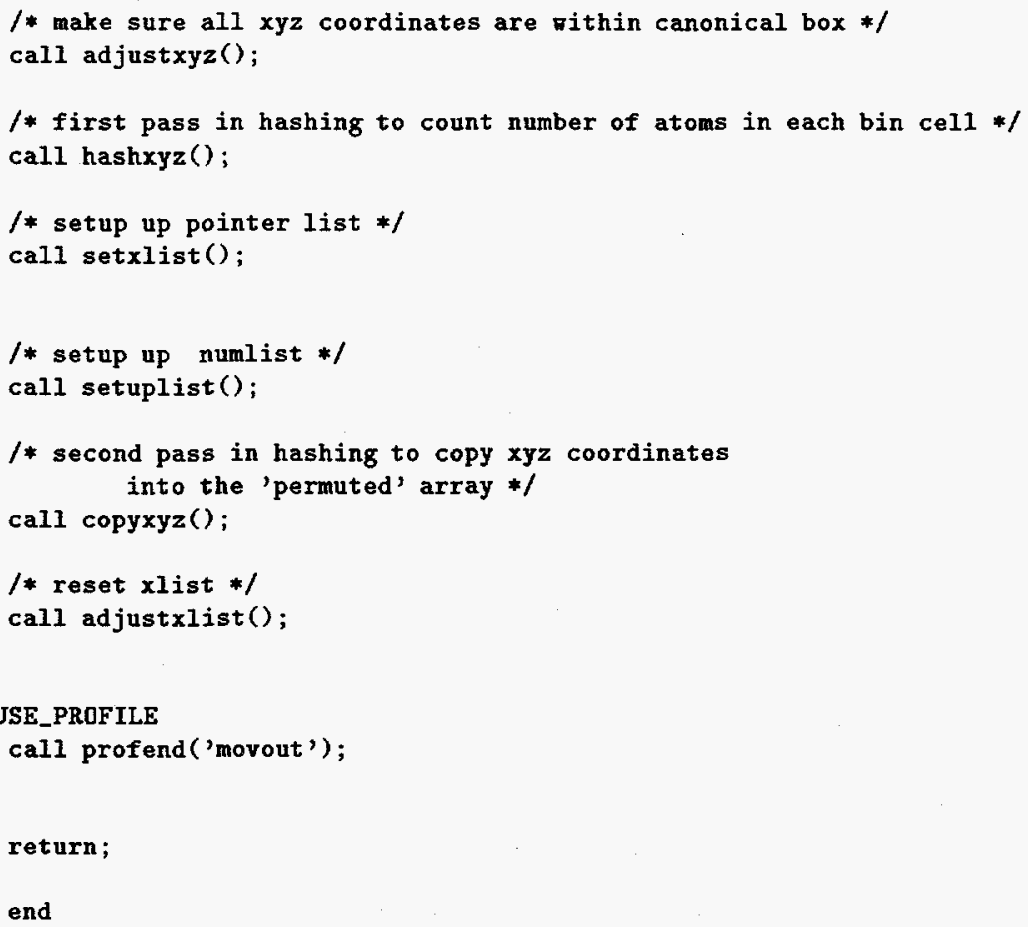

\section{2. subroutine adjustxyz}

*include "stdinc. $\mathrm{h}$ "

/* perform adjustment of $x y z$ to be rithin canonical box of $[x \min , x \max ] \times[y \min , y \max ] \times[z \min , z \max ] * \prime$

\#define logdev 16

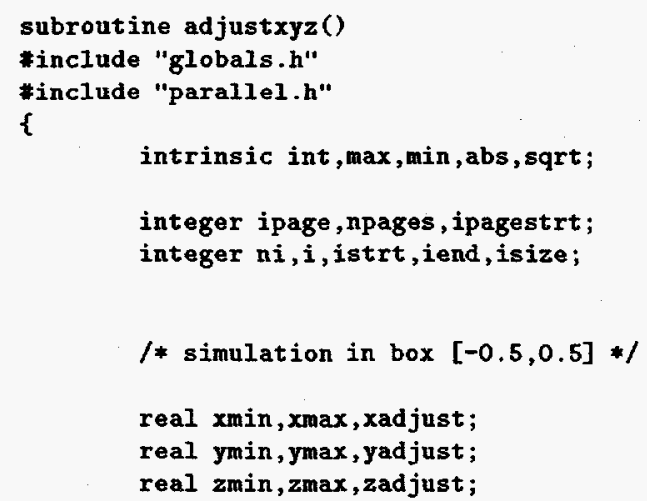


parameter $(x \min =-0.5, x \max =0.5, x \operatorname{adjust}=(x \max -x \min ))$; parameter $(y \min =-0.5, y \max =0.5$, yad just $=(y \max -y m i n))$; parameter $(\operatorname{zmin}=-0.5, \operatorname{zmax}=0.5, \operatorname{zadjust}=(\operatorname{zmax}-z \min ))$;

real one,zero;

parameter $($ one $=1.0$, zero $=0.0)$;

real xyz(3,pagesize);

tdefine $\mathrm{rx}(i) \mathrm{xyz}(1, i)$

*define ry(i) $x y z(2, i)$

Fdefine $x z(i)$ xyz $(3, i)$

logical isxok, isyok,iszok, isallok, isformatted;

$/ *==\pi== \pm==$ start execution $========* /$

*ifdef USE_PROFILE

call profstart('adjustxyz');

\#endif

call dogsync();

npages $=\operatorname{int}($ natoms $/$ pagesize $)+1$;

ipagestrt=myid;

call dodisable ( IGxyz ):

/* scatter operation */

call openrbuf (IGxyz, one, zero);

doloop4 (ipage, ipagestrt, npages, nproc) \{ istrt $=1+$ ipage*pagesize;

iend $=\min ($ natoms, istrt+pagesize-1);

isize = iend - istrt +1 ;

if (isize $<=0$ ) $\{$ break; \};

call donait (dobrgather( IGxyz, 3*isize, idxxyz (1,istrt), xyz));

if (IDEBUG $>=3$ ) $\ell$

تrite(logdev,*) 'adjustxyz: xyz before update'; arite (logdev, 9001) (istrt+(i-1), $r x(i), r y(i), r z(i), i=1, i s i z e)$;

9001 format $((1 x, i 6, ': ', 3(1 x, 1$ pe14.5)) )

\} ;

$\operatorname{doloop}(i, 1, i s i z e)($

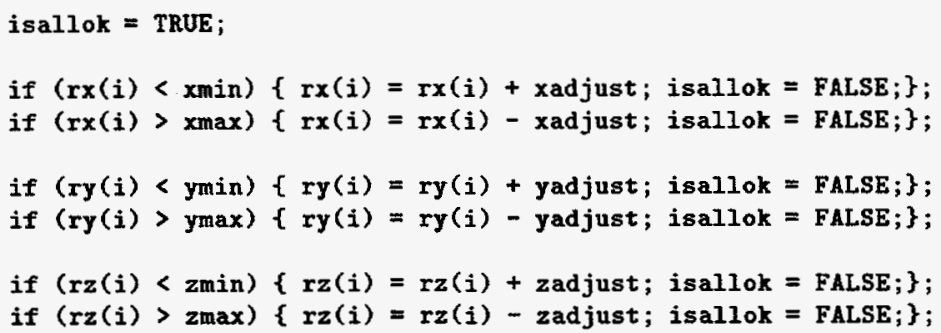




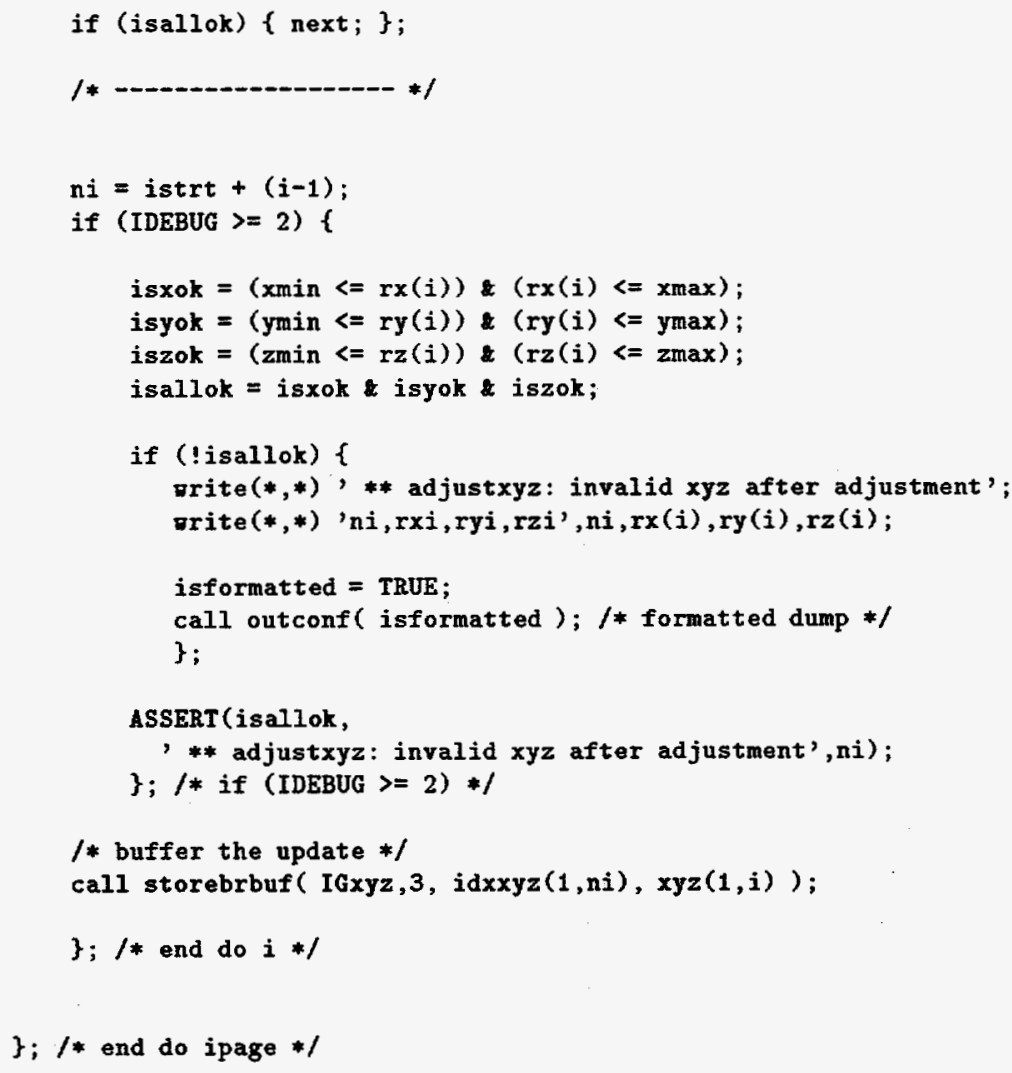

4.3. subroutine hashxyz

\#include "stdinc. $h$ "

/* perform hashing of $x y z$ to count number of atoms in each bin $* /$ *define USE_HCACHE 1 


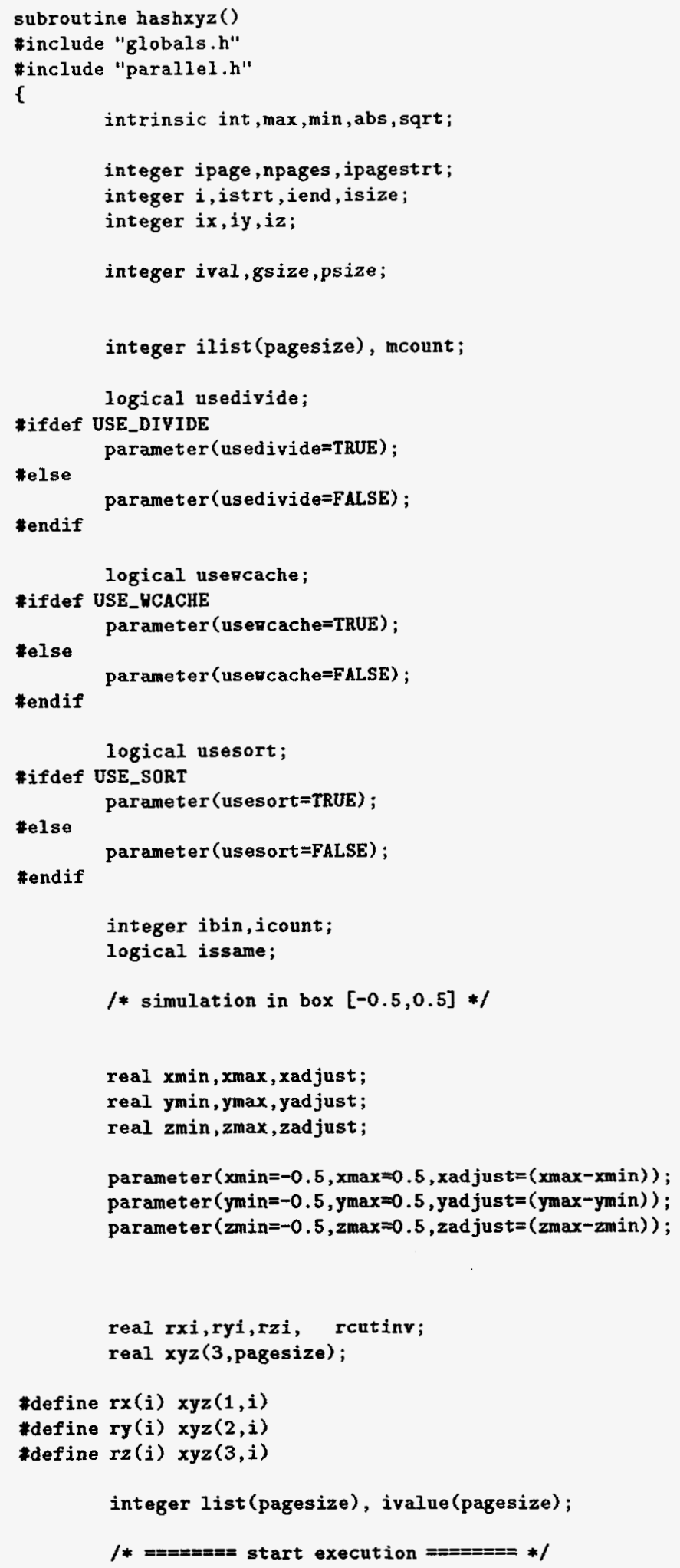




\author{
tifdef USE_PROFILE \\ call profstart ('hashxyz'); \\ *endif
}

call doenable ( IGxyz);

call dodisable ( IGncount);

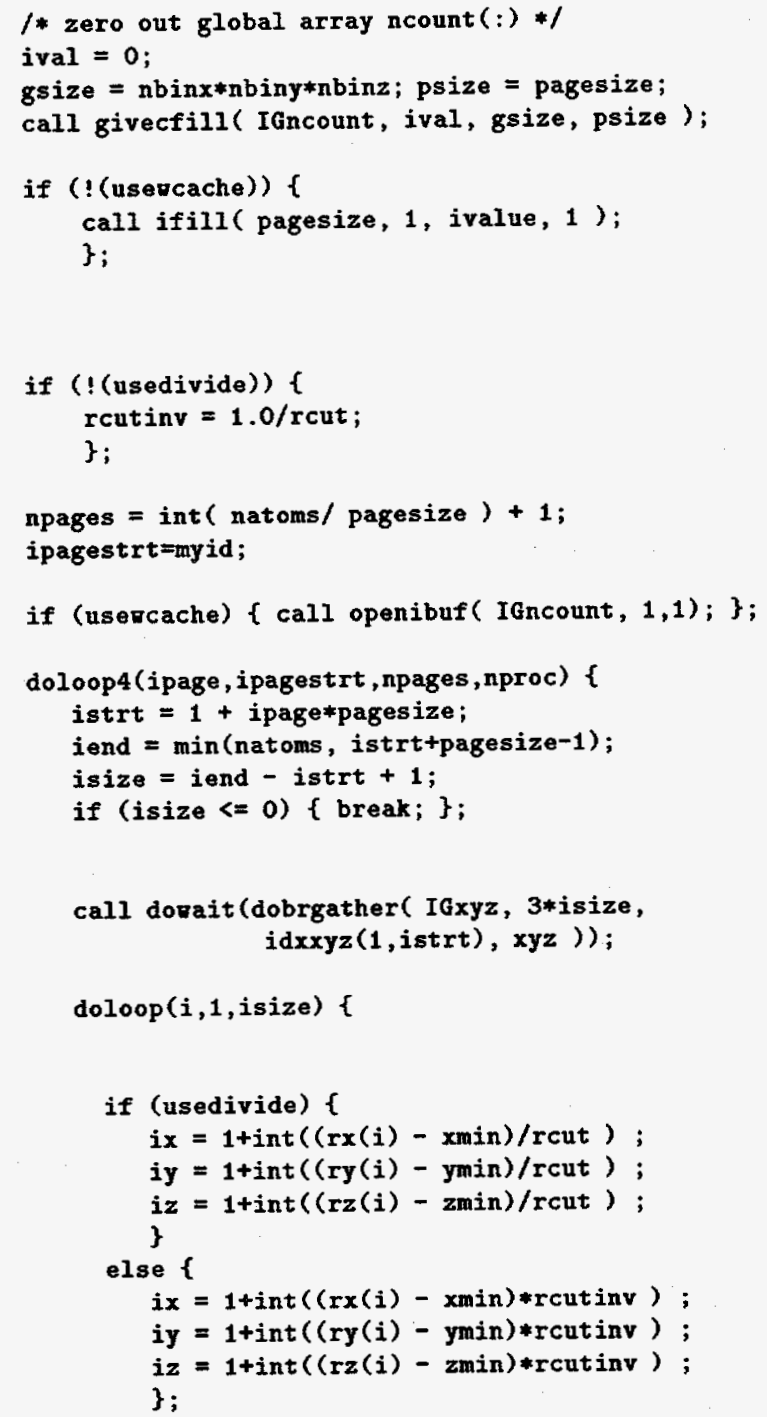




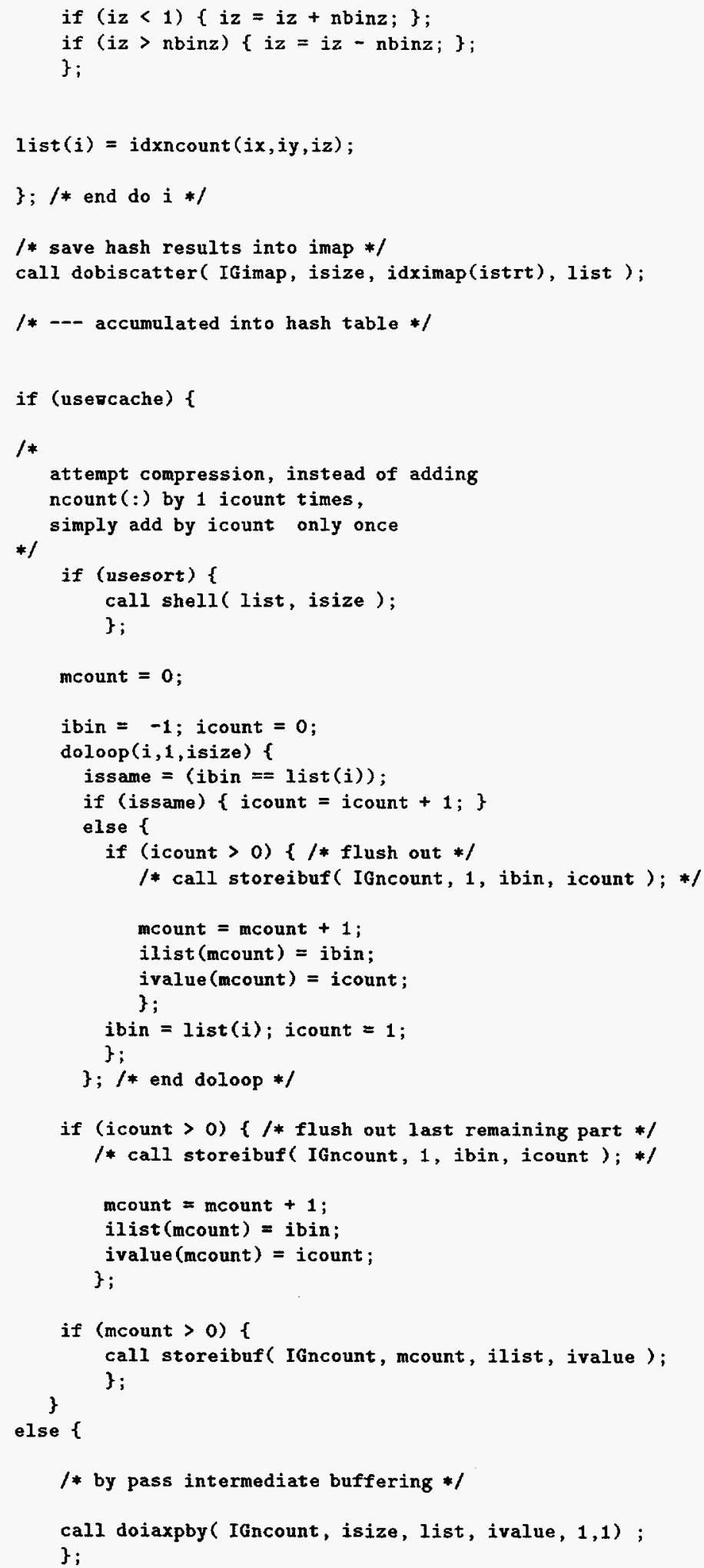




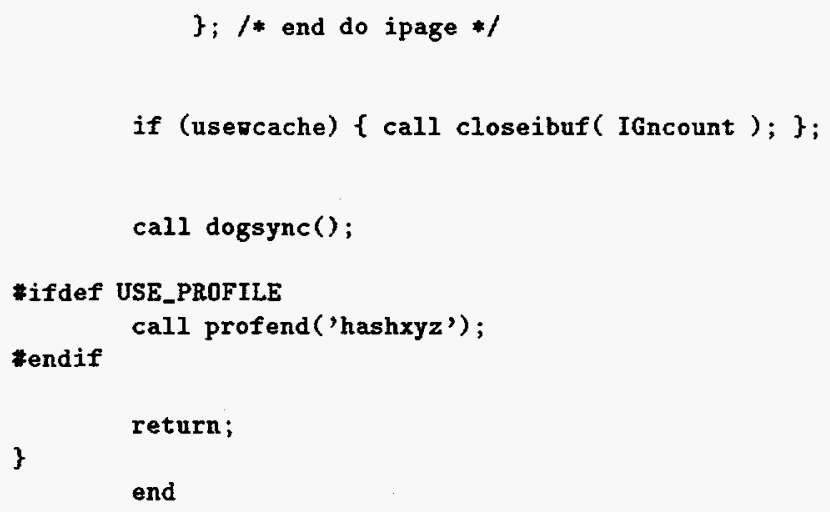




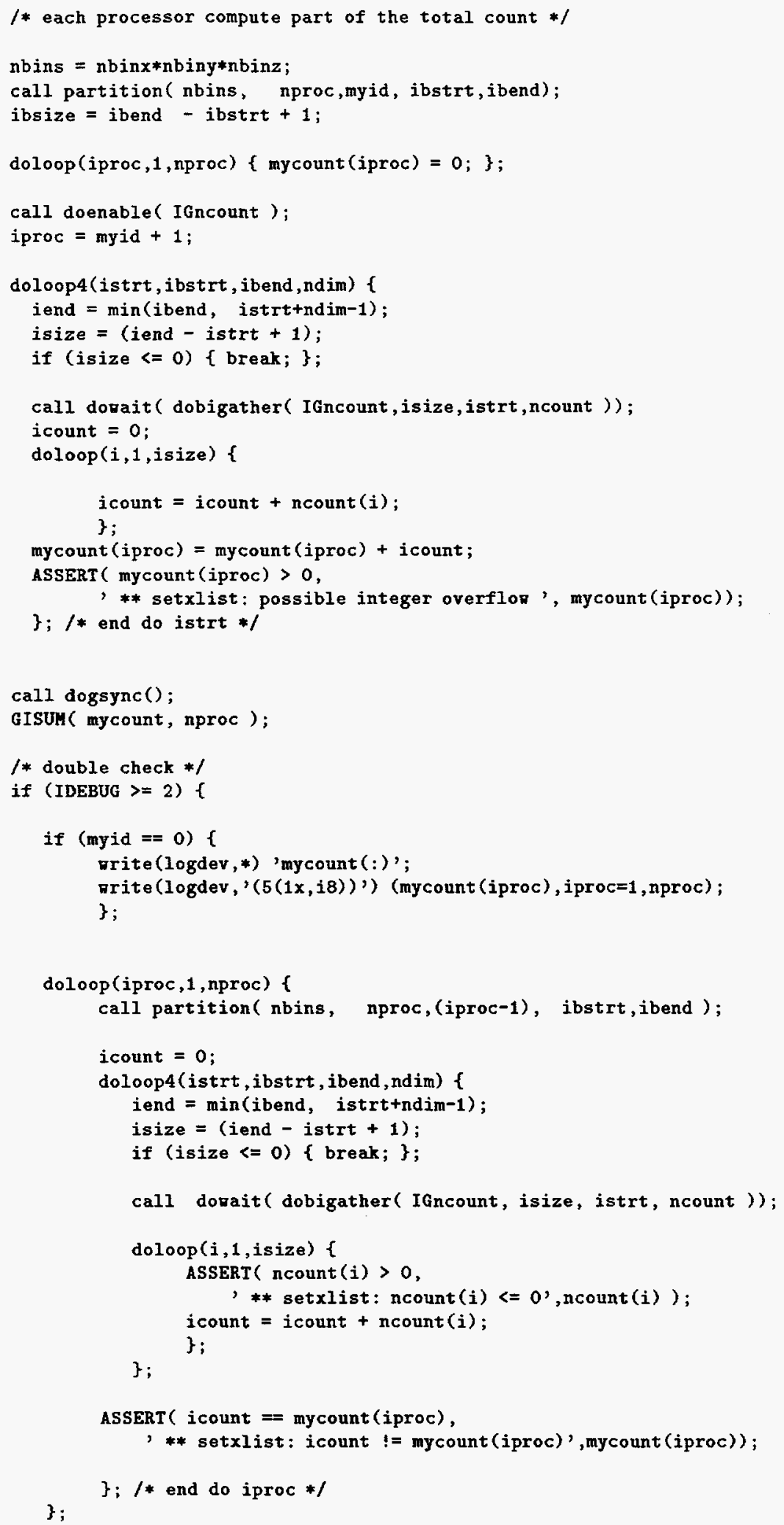




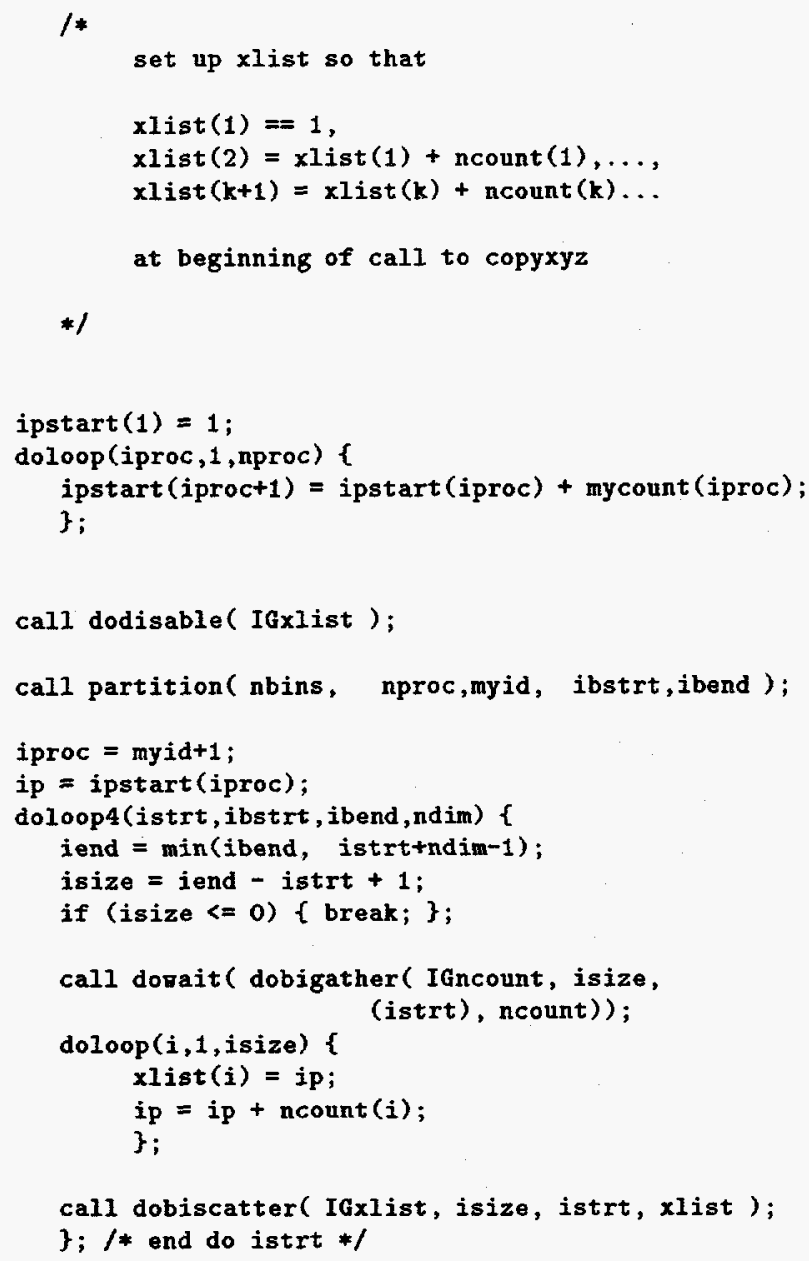

call dogsync();

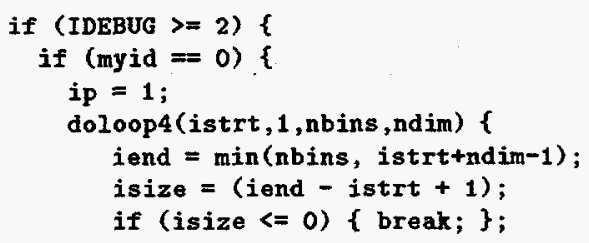




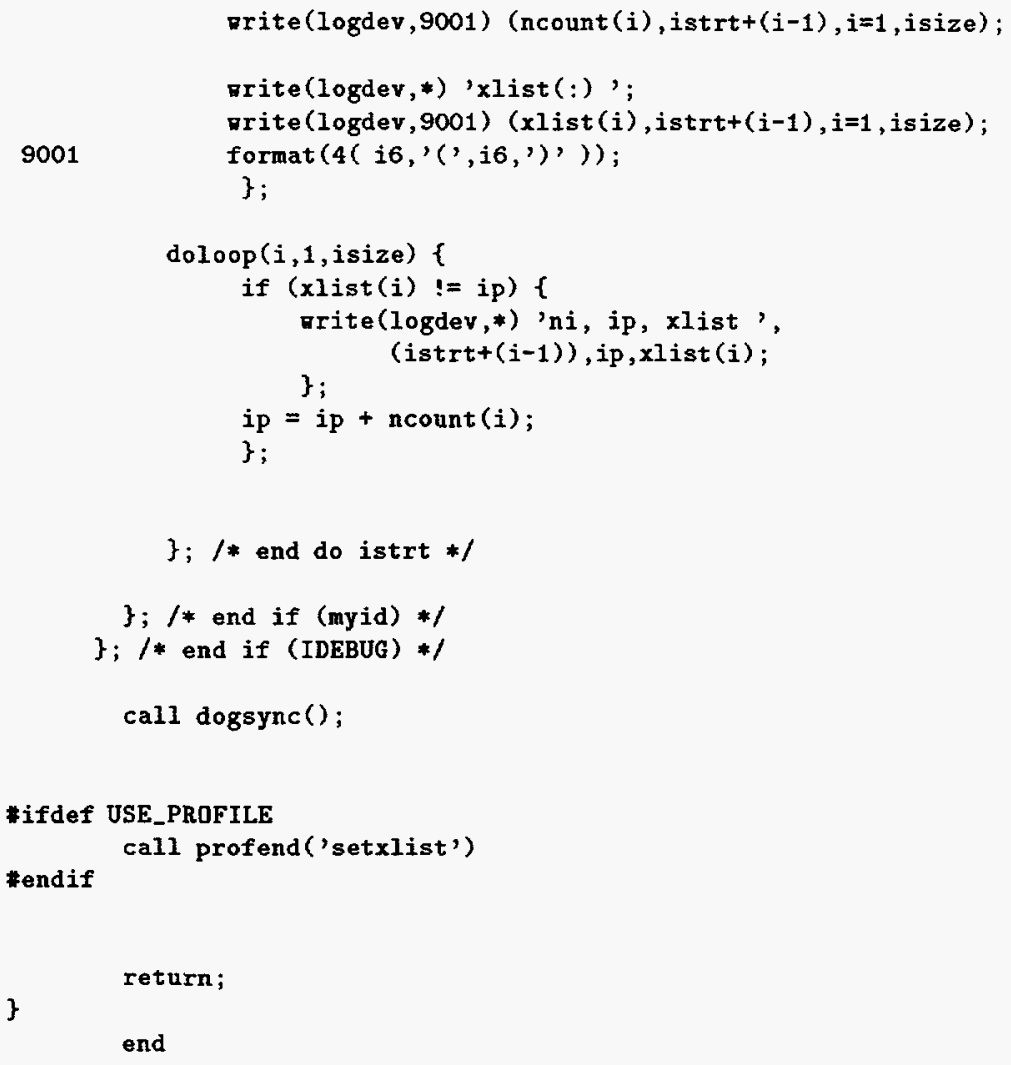

4.5. subroutine setuplist

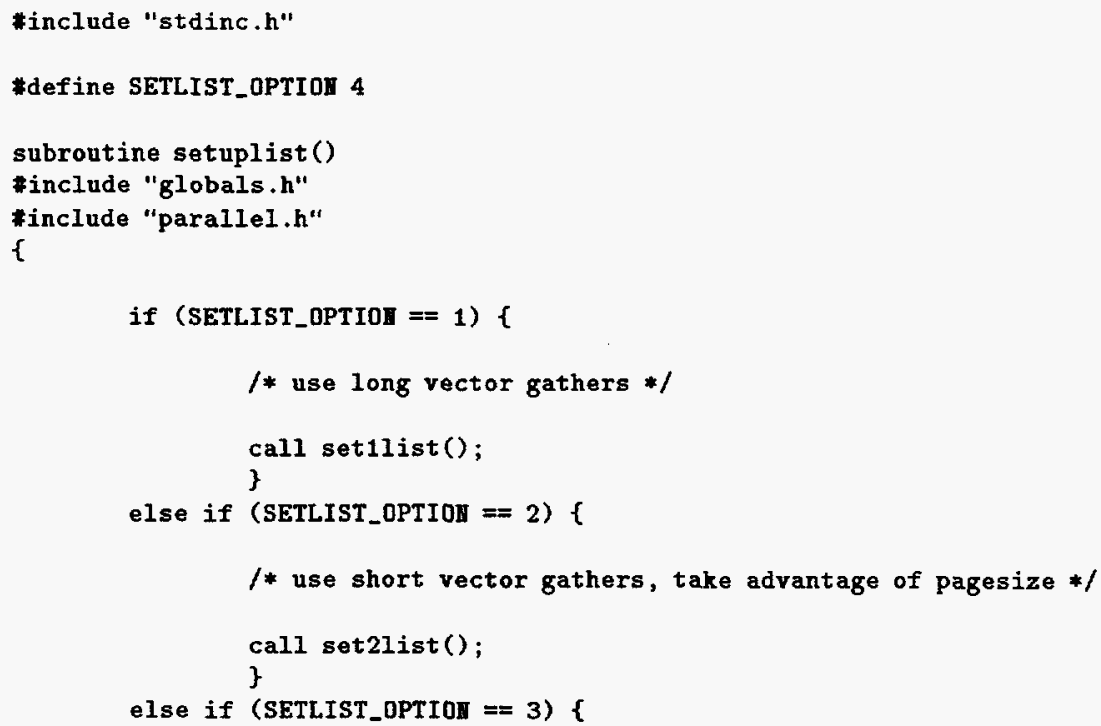




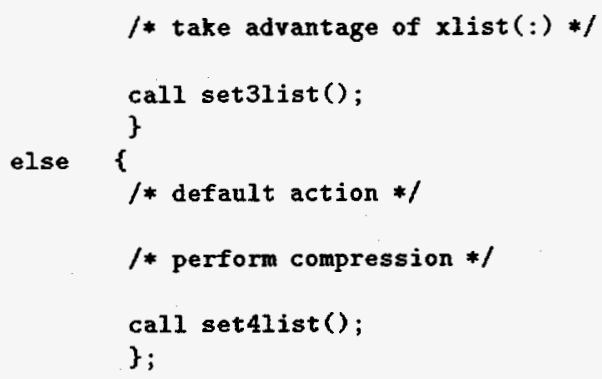

4.6. subroutine set4list

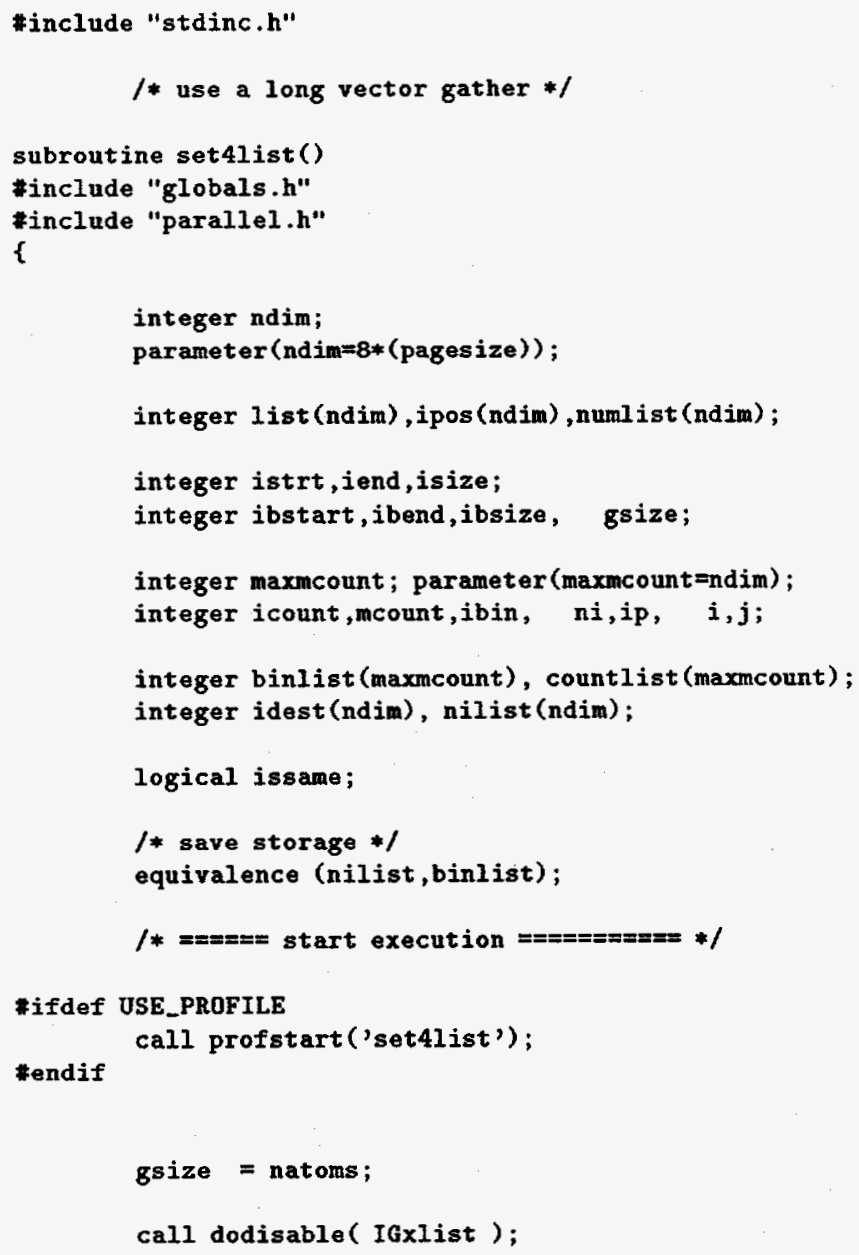




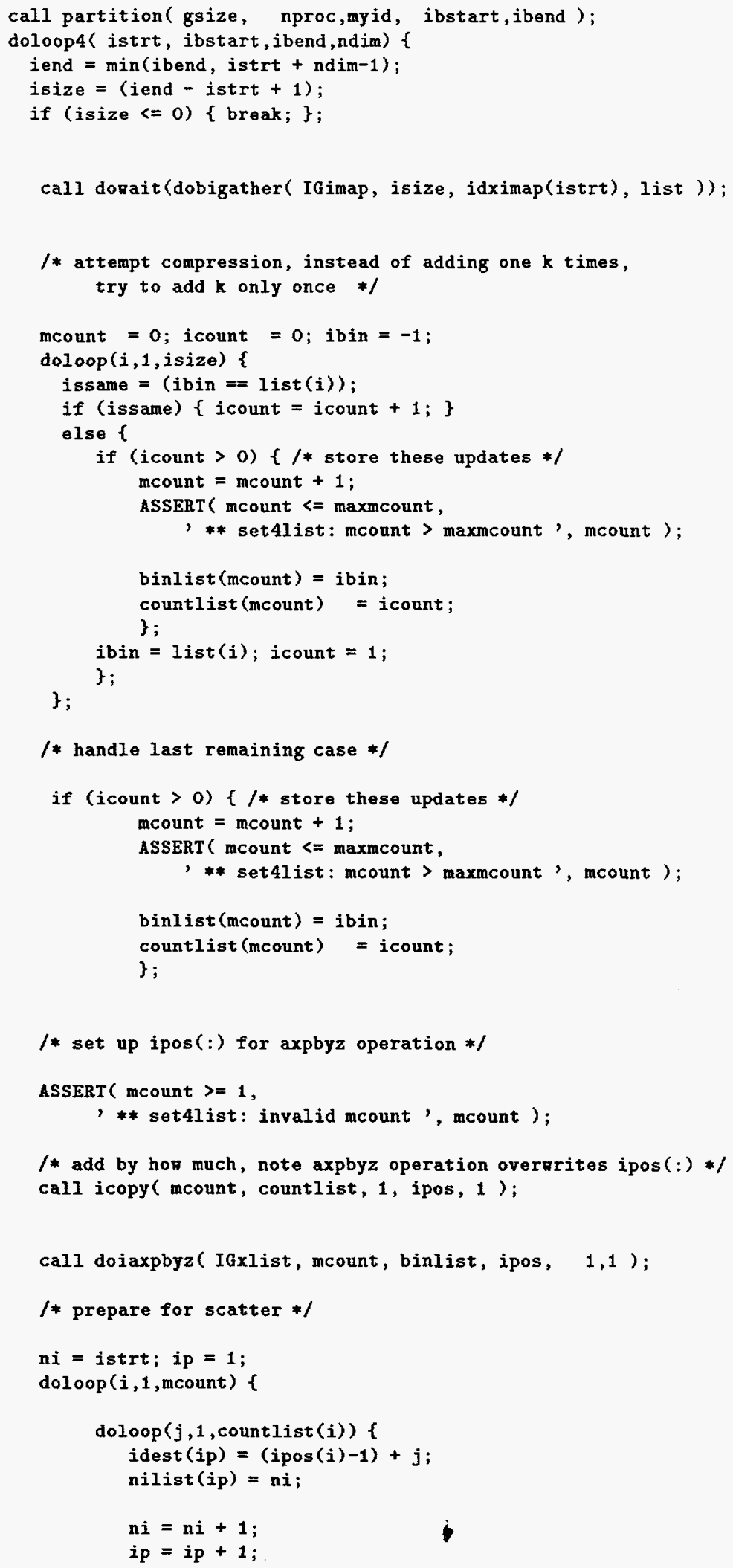




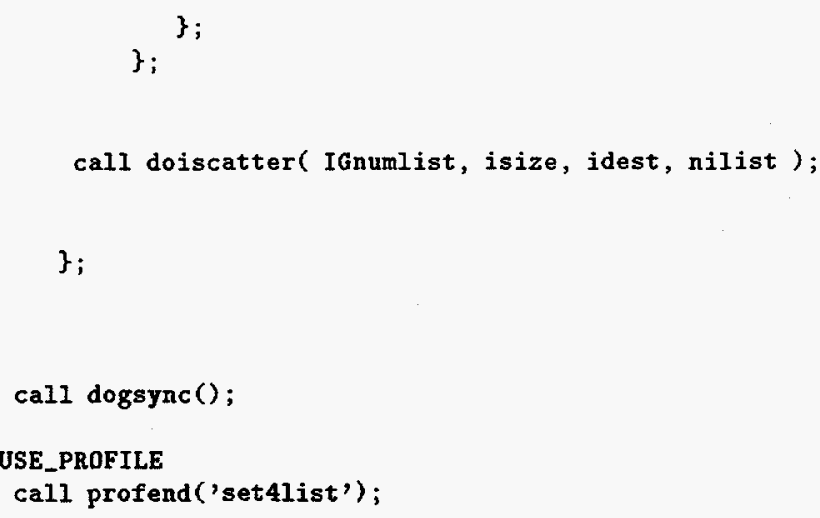

\section{7. subroutine copyxyz}

\#include "stdinc.h"

\#define logder 16

/* perform hashing of $x y z$ and copy coordinatest to new array *f

\#define ISWAP( $i a, i b)\{i t e m p=i a ; i a=i b ; i b=i t e m p ;\}$;

subroutine copyxyz()

\#include "globals.h"

\#include "parallel.h"

\{

logical usedfxyz;

\#ifdef USE_DFXYZ

parameter (usedf $x y z=T R U E$ );

*else

*endif

parameter (usedfxyz=FALSE);

STRIIG name; STRIrG ctype;

integer psize,gsize, blocksize;

integer ibstart,ibend; 


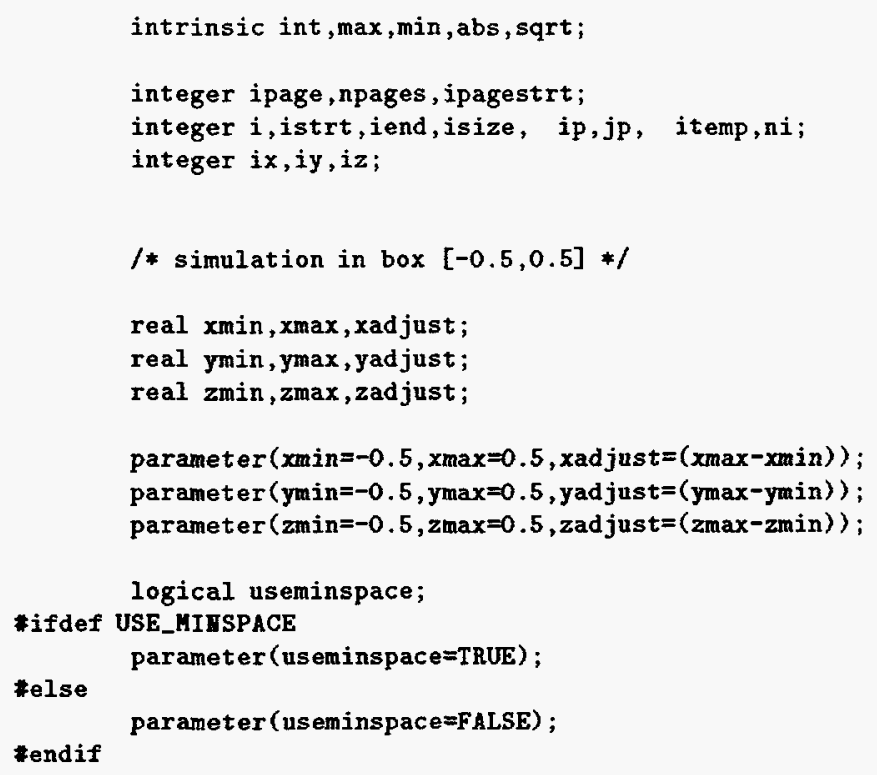

*define ndim pagesize

integer numlist (ndim);

integer iposxyz(3,ndim);

real xyz(3,ndim); real vxyz(3,ndim); equivalence (xyz,vxyz);

\#define $r x(i)$ xyz $(1, i)$

*define ry(i) xyz(2,i)

tdefine rz(i) xyz(3,i)

$/ *========$ start execution $========* /$

*ifdef USE_PROFILE

call profstart ('copyxyz')

*endif

/* disable everything */

call dodisable $(0)$;

if (useminspace) \{

$1 *$ free storage for imap and recreate fxyz *I

/* disable everything, extra safe, and 
encourage memory compaction $* /$

call dodisable $(0)$;

call dogsync();

call dodestroy ( IGimap);

call dogsync()

ctype = 'real' // char (0);

gsize $=3 *$ natoms; psize $=3 *$ pagesize $;$ blocksize $=1$;

name $=' f x y z(3$, natoms $), / / \operatorname{char}(0)$;

call dodeclare ( IGfxyz, name, gsize, ctype, psize, blocksize);

call dogsync();

\};

/* use a "pull" strategy to copy the data */

*ifdef USE_PROFILE

\#endif

cal1 profstart ('copyxyz:cp xyz');

/* should be local, no need to search cache */

call dodisable ( IGnumlist);

call doenable( IGxyz);

call dodisable (IGfxyz).

/* permuted copy xyz to fxyz, then fxyz to xyzlist *f

gsize = natoms;

npages $=\operatorname{int}($ gsize/ pagesize $)+1$;

ipagestrt=myid;

doloop4 (ipage, ipagestrt, npages, nproc) \{

istrt $=1$ + ipage*pagesize;

iend $=\min ($ gsize, istrt+pagesize -1 );

isize $=$ iend - istrt +1

if (isize $<=0$ ) $\{$ break; $\}$;

call dorait ( dobigather( IGnumlist, isize, idxnumlist (istrt), numlist ));

$\operatorname{doloop}(i, 1$, isize $)\{$

ni $=$ numlist $(i)$;

$j p=i d x x y z(1, n i)$;

/* assume continugous storage $* /$

$i p o s x y z(1, i)=j p$

$i \operatorname{posxyz}(2, i)=j p+1$;

$i p o s x y z(3, i)=j p+2$;

\}

call dorgather (IGxyz, 3*isize, iposxyz, xyz);

call dobrscatter (IGfxyz, $3 *$ isize, idxfxyz (1,istrt), xyz); 
\}; $/$ * end do ipage *

\#ifdef USE_PROFILE

\#endif

call profend('copyxyz:cp xyz');

call dogsync();

/*

safer to copy vectors than renaming them, vill simply suap

$*$ /

global array descriptors to rename them if this is really costly.

call dodisable ( IGxyzlist);

gsize $=3 *$ natoms $;$ psize $=3 *$ pagesize;

call grveccopy (IGfxyz, gsize, psize, IGxyzlist);

call dogsyne();

/* permuted copy vxyz to fxyz, then fxyz to vxyz *I

call doenable ( IGvxyz);

call dodisable (IGfxyz);

\#ifdef USE_PROFILE

\#endif

call profstart ('copyxyz:cp vel');

gsize = natoms;

npages $=\operatorname{int}($ gsize $/$ pagesize $)+1 ;$

ipagestrt=myid;

doloop4 (ipage, ipagestrt, npages, nproc) f

istrt $=1+$ ipage*pagesize;

iend $=\min ($ gsize, istrt+pagesize-1);

isize $=$ iend - istrt +1 ;

if (isize $<0$ ) $\{$ break; $\}$;

call dorait ( dobigather( IGnumlist, isize, idxnumlist (istrt), numlist ));

doloop $(i, 1$, isize $)\{$

ni $=$ numlist $(i)$;

$j p=i d x x y z(1, n i)$;

/* assume continugous storage $* /$

$\operatorname{iposxyz}(1, i)=j p ;$

$i \operatorname{posxyz}(2, i)=j p+1$

$i \operatorname{pos} x y z(3, i)=j p+2$;

\};

call dorgather( IGvxyz, 3*isize, iposxyz, vxyz);

call dobrscatter( IGfxyz, $3 *$ isize, idxfxyz(1,istrt), vxyz);

\}; $/ *$ end do ipage *1

\#ifdef USE_PROFILE

\#endif call profend('copyxyz:cp vel');

call dogsync(); 


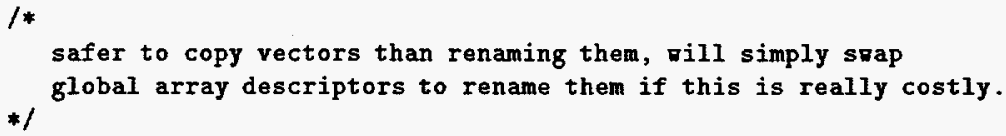

\section{8. subroutine force}

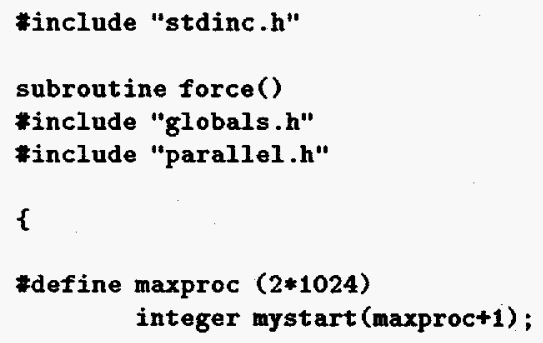


integer istrt,iend, ncols, binsize;

logical islast;

integer gsize,psize;

real one,zero;

parameter (one $=1.0$, zero $=0.0)$;

real8 oned,zerod;

parameter (oned $=1.0 \mathrm{~d} 0$, zerod $=0.0 \mathrm{~d} 0$ );

real rvalue;

reals dvalue;

/* perform dynamic load balancing and all force computations */

$f *========$ start execution $======* /$

\#ifdef USE_PROFILE

call profstart('force')

*endif

$\operatorname{ASSERT}($ ( $1<=$ nproc) \& (nproc $<=$ maxproc),

, ** force: invalid nproc', nproc);

\# ifdef IO_BALAICE

/* no need to call "calqork" */

call Ibalance( mystart);

\#else

call profstart('force:calrork');

call calwork();

call lbalance (mystart);

\#endif

call profend('force:calwork');

istrt = mystart $($ myid+1);

/* iend is the last column actually computed */

islast $=(($ myid +1$)==$ nproc $)$;

if (islast) \{

binsize $=$ nbiny*nbinz

iend $=$ binsize ;

子

else \{

iend $=$ mystart $((m y i d+1)+1)-1 ;$

\};

ncols $=($ iend - istrt +1$)$;

/* clear out array for accumulation */

gsize $=3 *$ natoms; psize $=3 *$ pagesize;

\#ifdef USE_DFXYZ

dvalue $=0.0$

call gdvecfill( IGfxyz, dvalue, gsize, psize);

*else

rvalue $=0.0$;

call grvecfill ( Iofxyz, rvalue, gsize, psize);

\#endif 


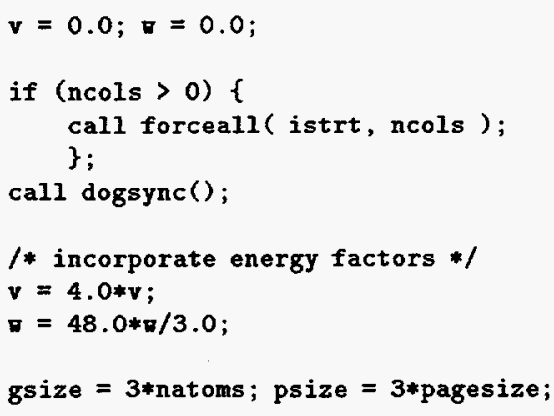

\section{9. subroutine forceall}

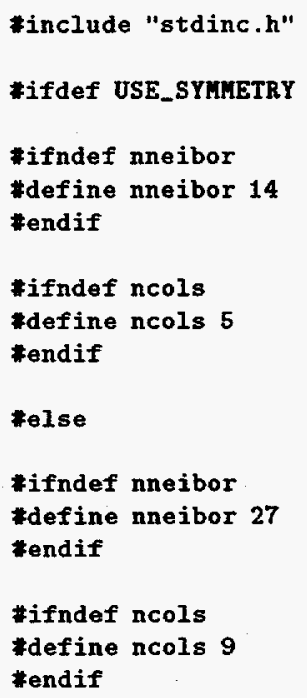




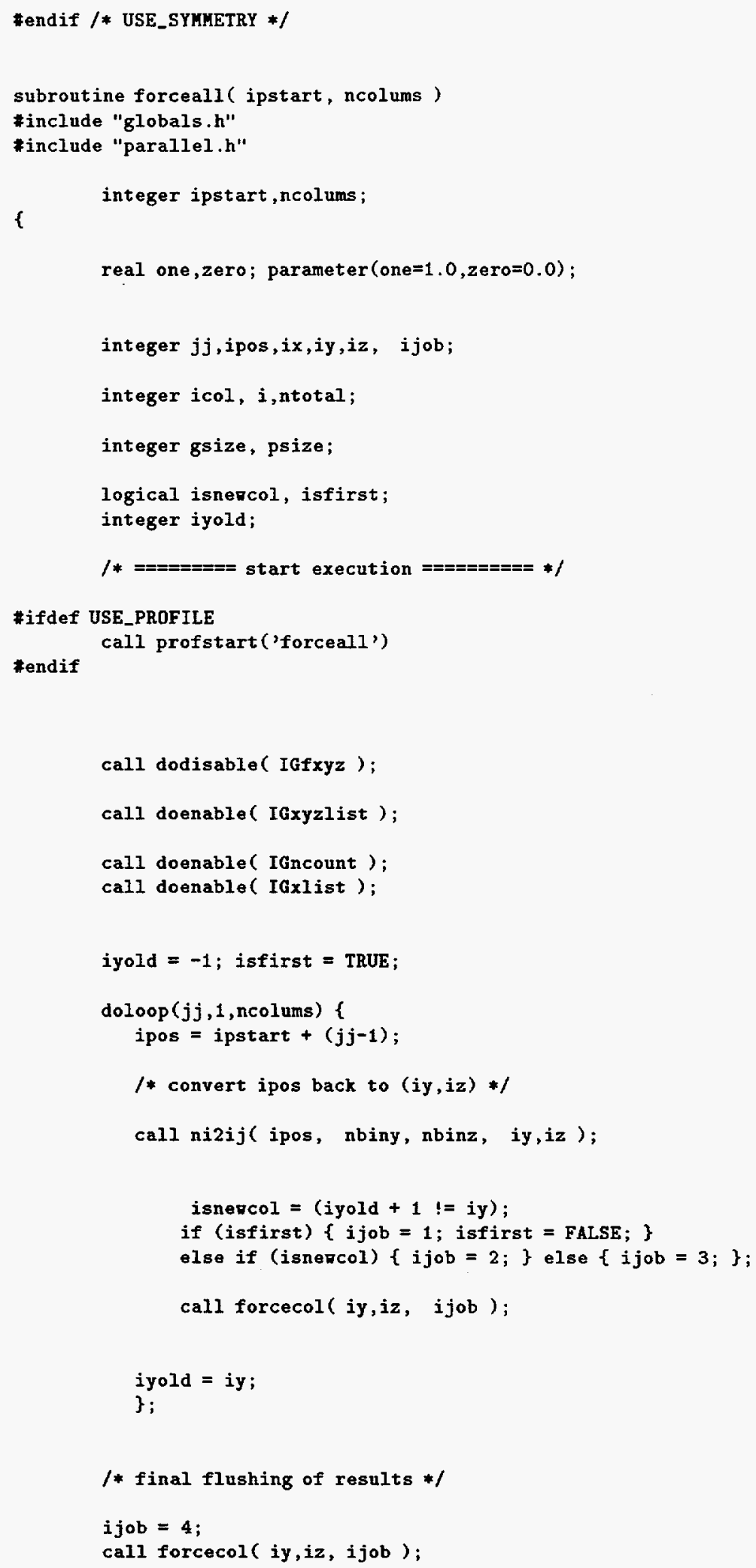




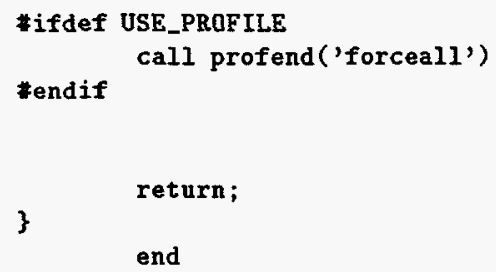

4.10. subroutine forcecol

*include "stdinc.h"

*undef USE_PROFILE

\#define logdev 16

*define eps $1.0 e-5$

*define isapprox $(r 1, r 2)(\operatorname{abs}((r 1)-(r 2))<=\operatorname{eps} * \max ($ one, $\max (\operatorname{abs}(r 1), \operatorname{abs}(r 2))))$

*define RETURI_LABEL 999

*ifdef USE_SYMMETRY

*ifndef nneibor

*define nneibor 14

*endif

*ifndef ncols

*define ncols 5

*endif

*else

*ifndef nneibor

*define nneibor 27

*endif

*ifndef ncols

*define ncols 9

*endif

*endif /* USE_STHMETRY * $/$

*define CHECRXYZ( icol) \{ 1

doloop $(j x, 1$, nbinx $)\{1$

1

call doigather ( IGncount, 1, idxncount $(j x, j y, j z)$, icount); I ASSERT $($ icount $==$ ncount $(j \mathrm{x}, i \operatorname{col}), \mid$

'** forcecol: icount != ncount $(j x, i c o l)$, icount); 
1

1

$j p=x l i s t(i c o l) ; \quad 1$

$\operatorname{doloop}(j, 1, j x-1)\{j p=j p+n \operatorname{count}(j, i c o l) ;\} ;$

call doigather (IGxlist, 1 , idxxlist $(j x, j y, j z)$, ip ) ; 1 ASSERT $($ ip $==j p, \mid$

' ** forcecol: error in xlist calculation', jp ); 1

1

if (icount $>0)\{1$

call domait ( dobrgather (IGxyzlist, 3*icount, ।

1 idxxyzlist $(1, i p)$, txyz )); 1

1

doloop $(j, 1$, icount $)\{\backslash$

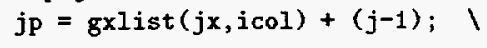

1

\}; $/ *$ end do $j x * / \quad 1$

\};

*define FOLDBC $(i c o l)\{1$

1

$1 *$ fold boundary contribution back */ 1

isrc $=\operatorname{gxlist}(0$, icol $) ;$ idest $=\operatorname{gxlist}($ nbinx, icol $) ; 1$

icount $=\operatorname{ncount}(0$, icol $) ;$

doloop(i,1,icount) $\{1$

$f x($ idest $)=f x($ idest $)+f x($ isrc $) ; 1$ $f y($ idest $)=f y($ idest $)+f y($ isrc $) ; 1$

1 $f z($ idest $)=f z($ idest $)+f z($ isrc $) ; 1$

1

idest $=$ idest $+1 ;$ isrc $=$ isrc $+1 ; \quad \mid$ \}$; 1$

isrc $=\operatorname{gxlist}(n b i n x+1$, icol $) ;$ idest $=\operatorname{gxlist}(1$, icol $) ; \quad$

1 icount $=n \operatorname{count}(n b i n x+1, i c 01) ; \quad 1$

doloop (i,1,icount $) \in \backslash$ $f x($ idest $)=f x($ ides $t)+f x($ isrc $) ; \quad 1$ $f y($ idest $)=f y$ (idest $)+f y($ isrc $) ; ।$

1 $f z($ ides $t)=f z($ idest $)+f z($ isrc $) ; 1$

1 idest $=$ idest $+1 ;$ isrc $=$ isrc $+1 ; \quad \mid$ \}; 1

\};

\#define FLUSHOUT( $i c o l)\{1$

if (ntotal (icoI) >0) \{

FOLDBC (icol); 1

1

if (usedfxyz) $\{1$

call dobdaxpby (IGfxyz, $3 *$ ntotal(icol), ।

idxfxyz $(1, x l i s t(i c 01)), \mid$

\} 1

fxyz(1, gxlist (1, icol)), oned, oned); ।

else $\{1$ 


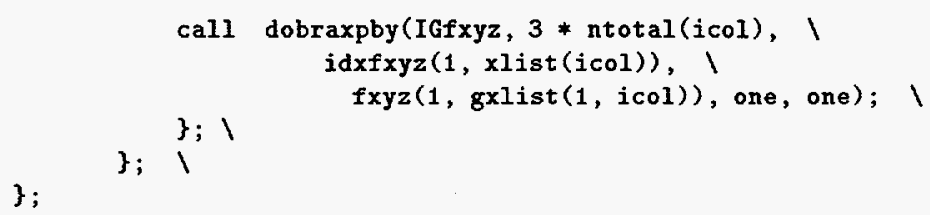

\} 


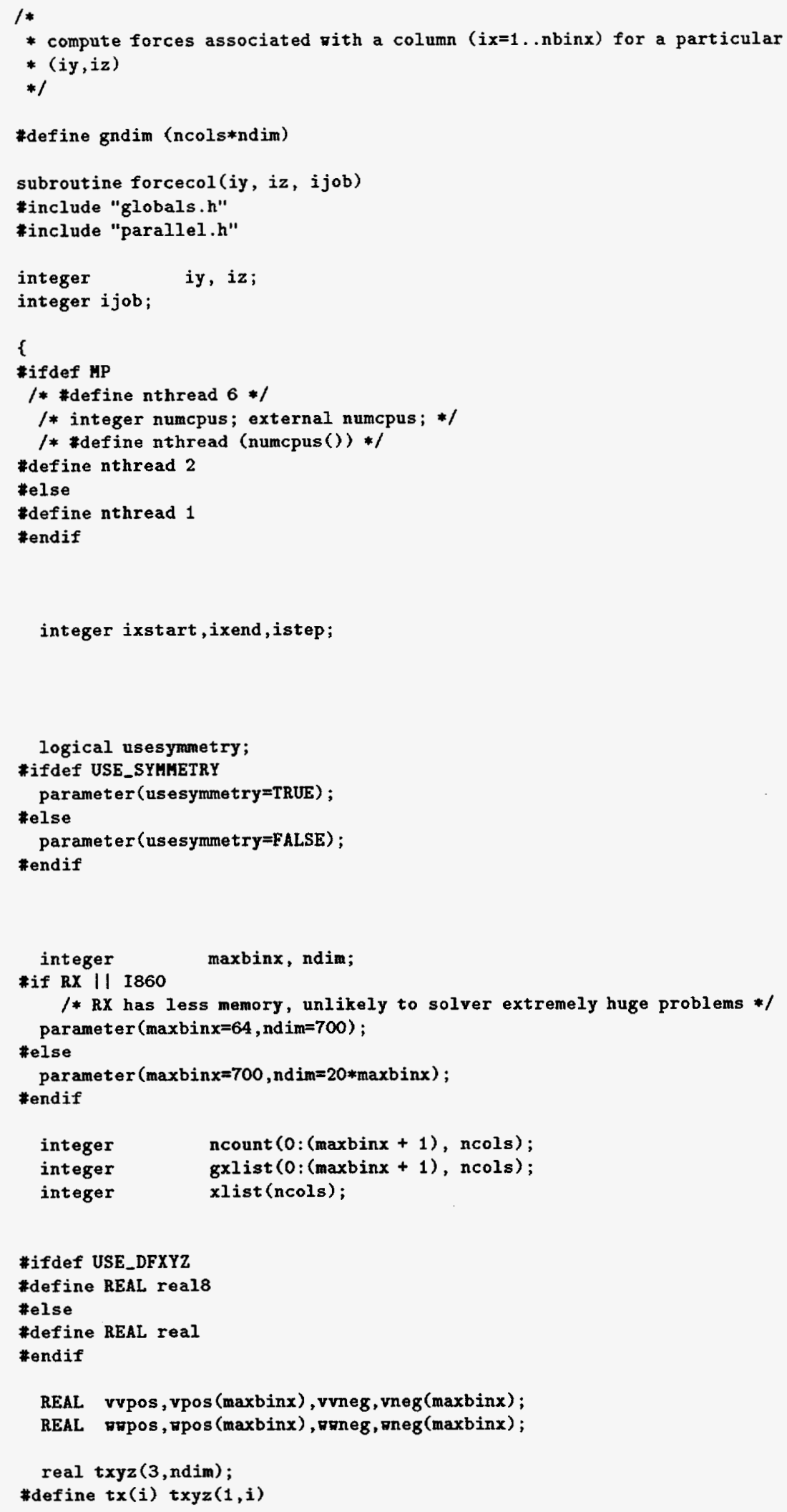




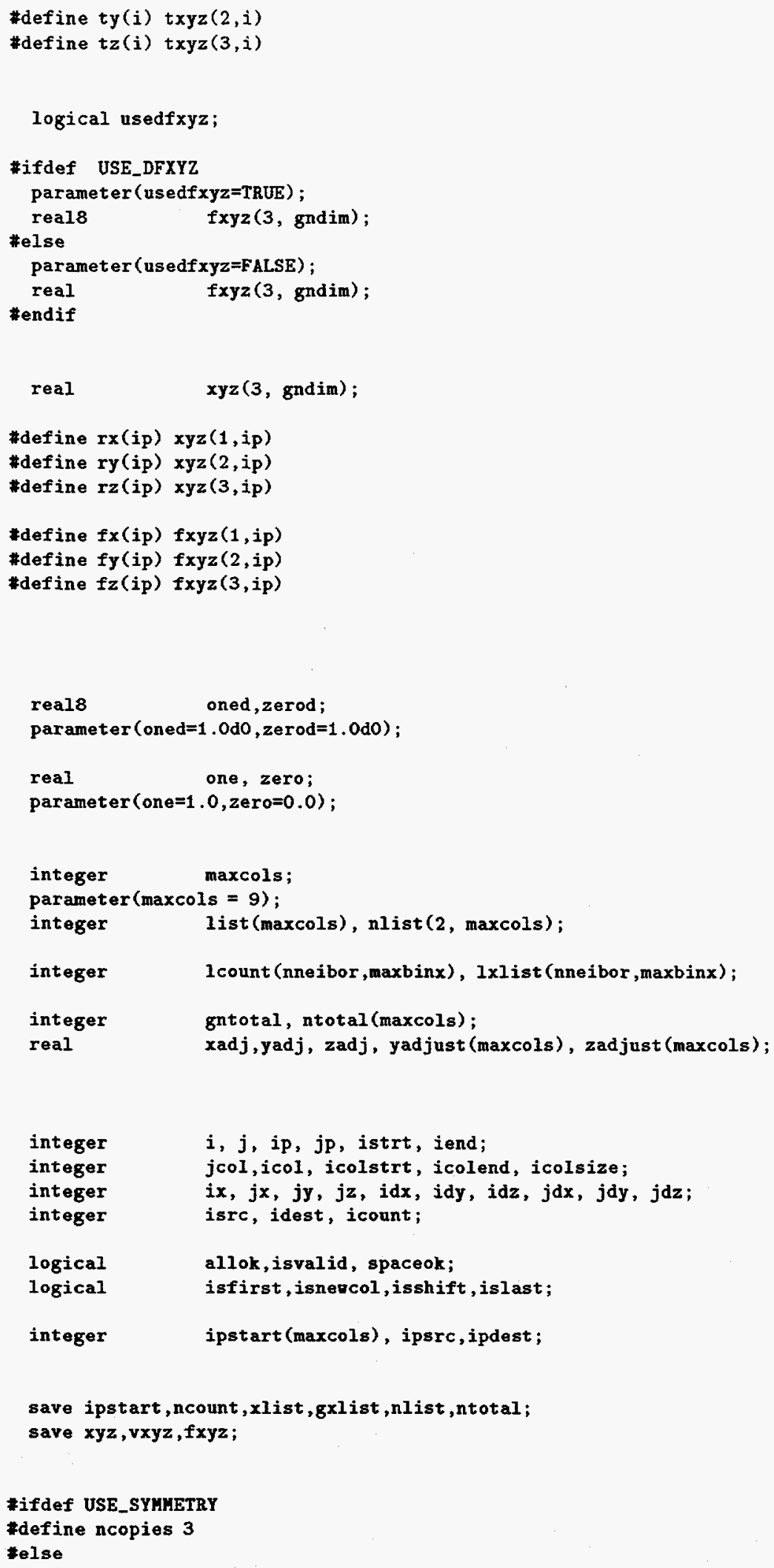




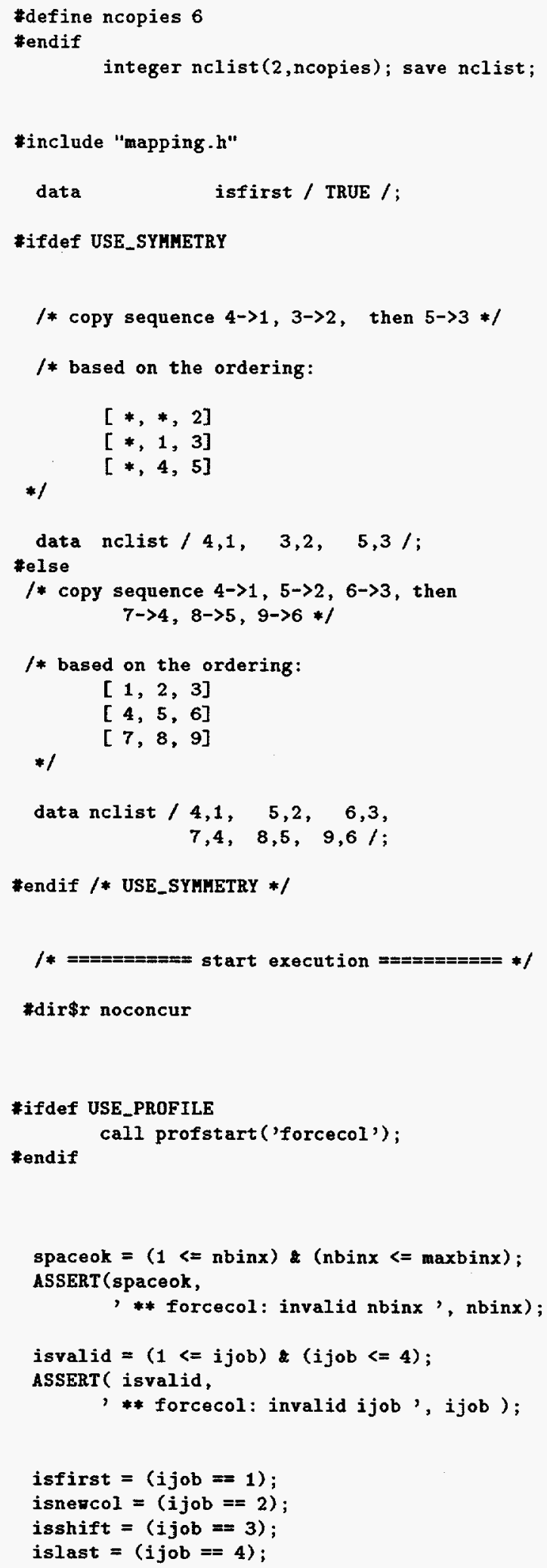




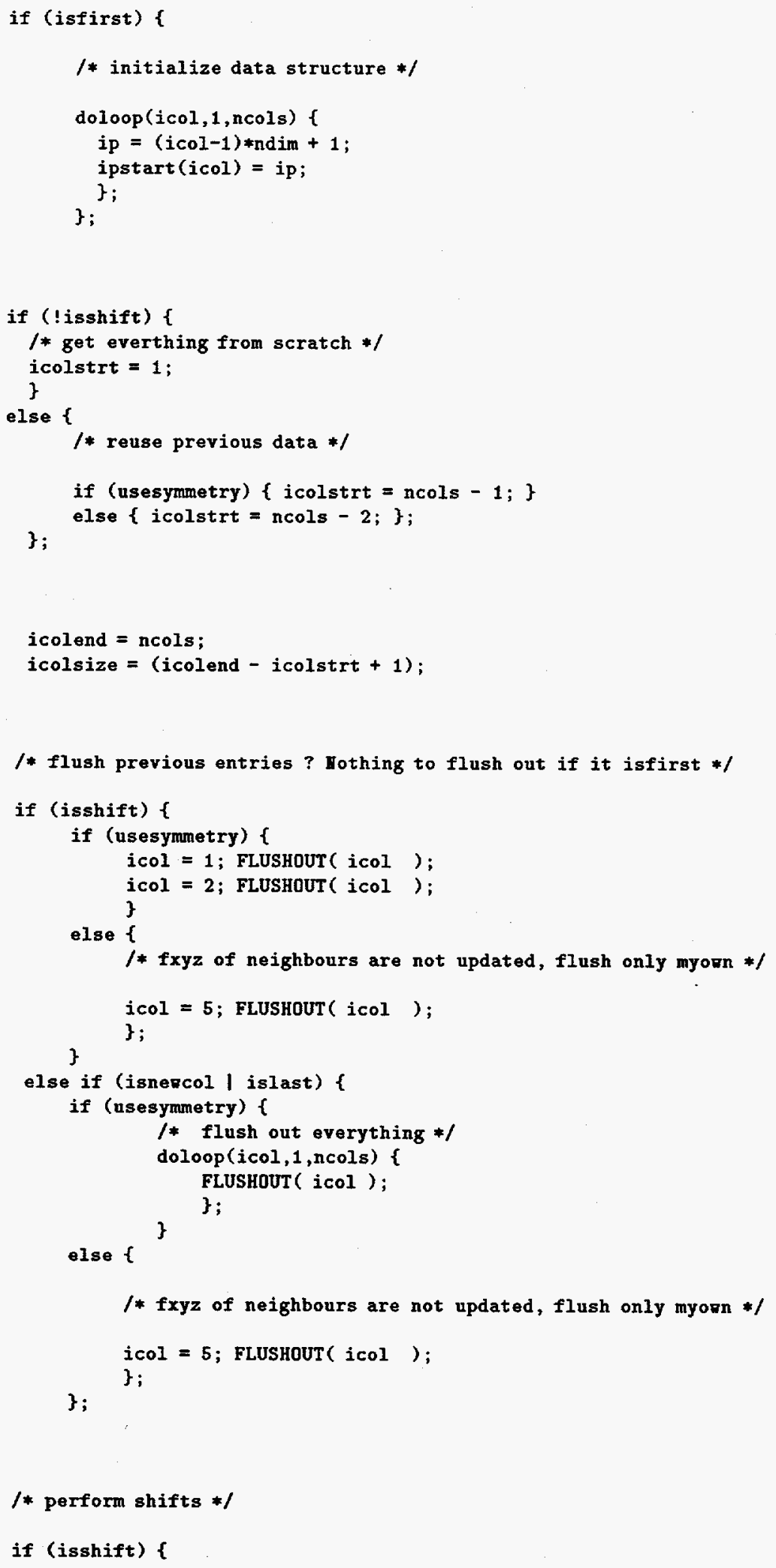




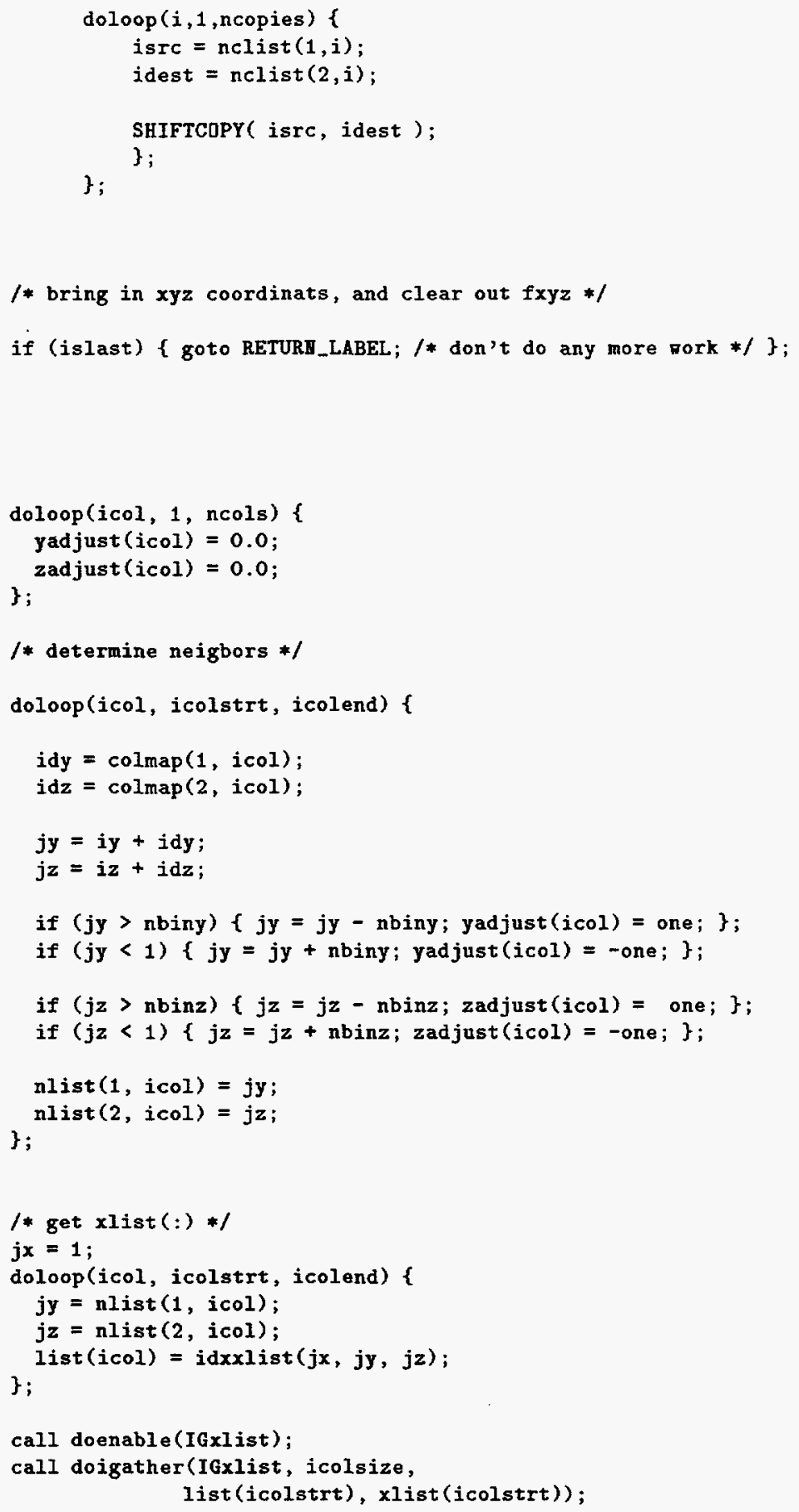

/* get ncount(:) for colums *I

call doenable( IGncount);

doloop (icol, icolstrt, icolend) \{ 


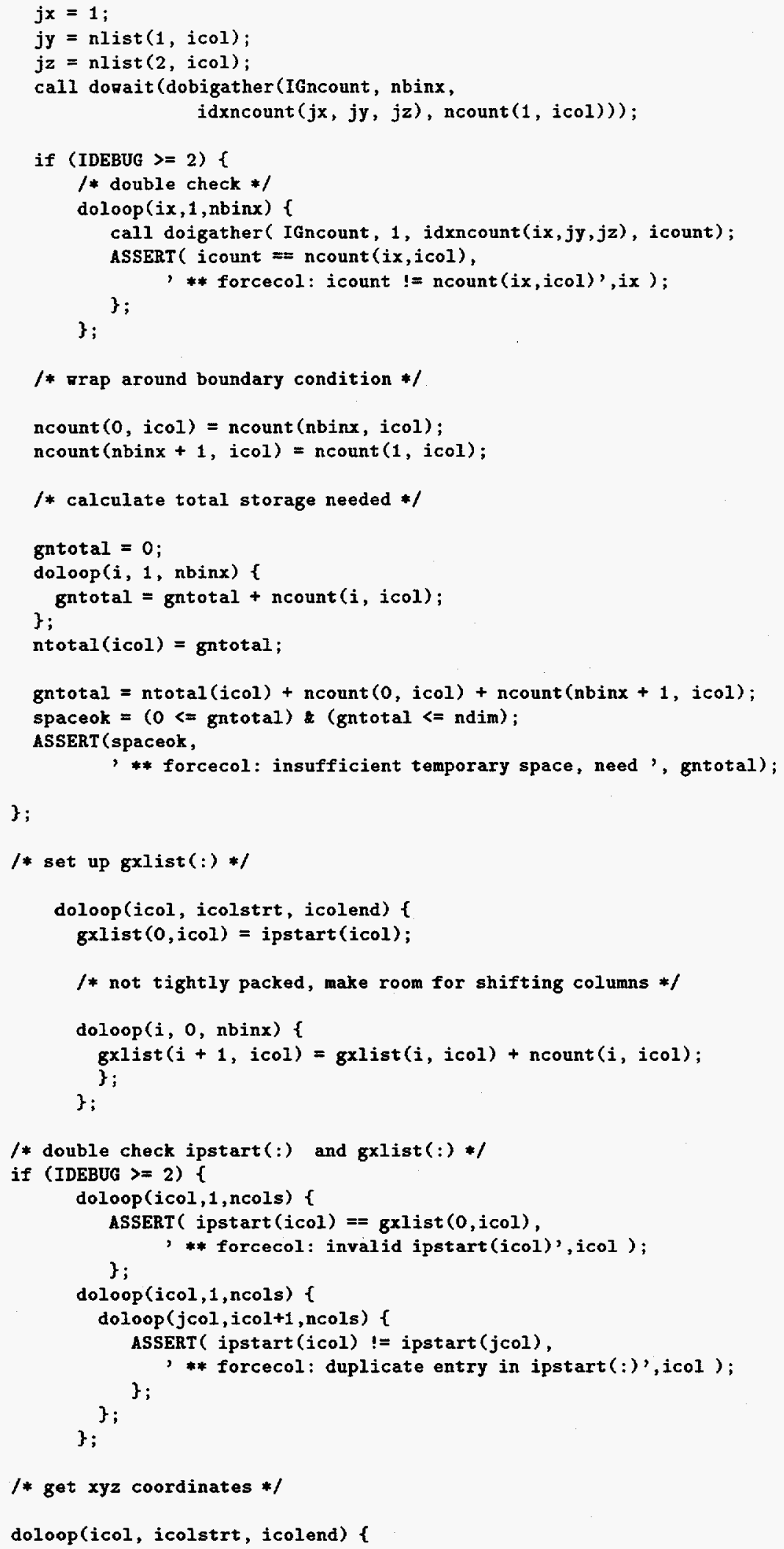




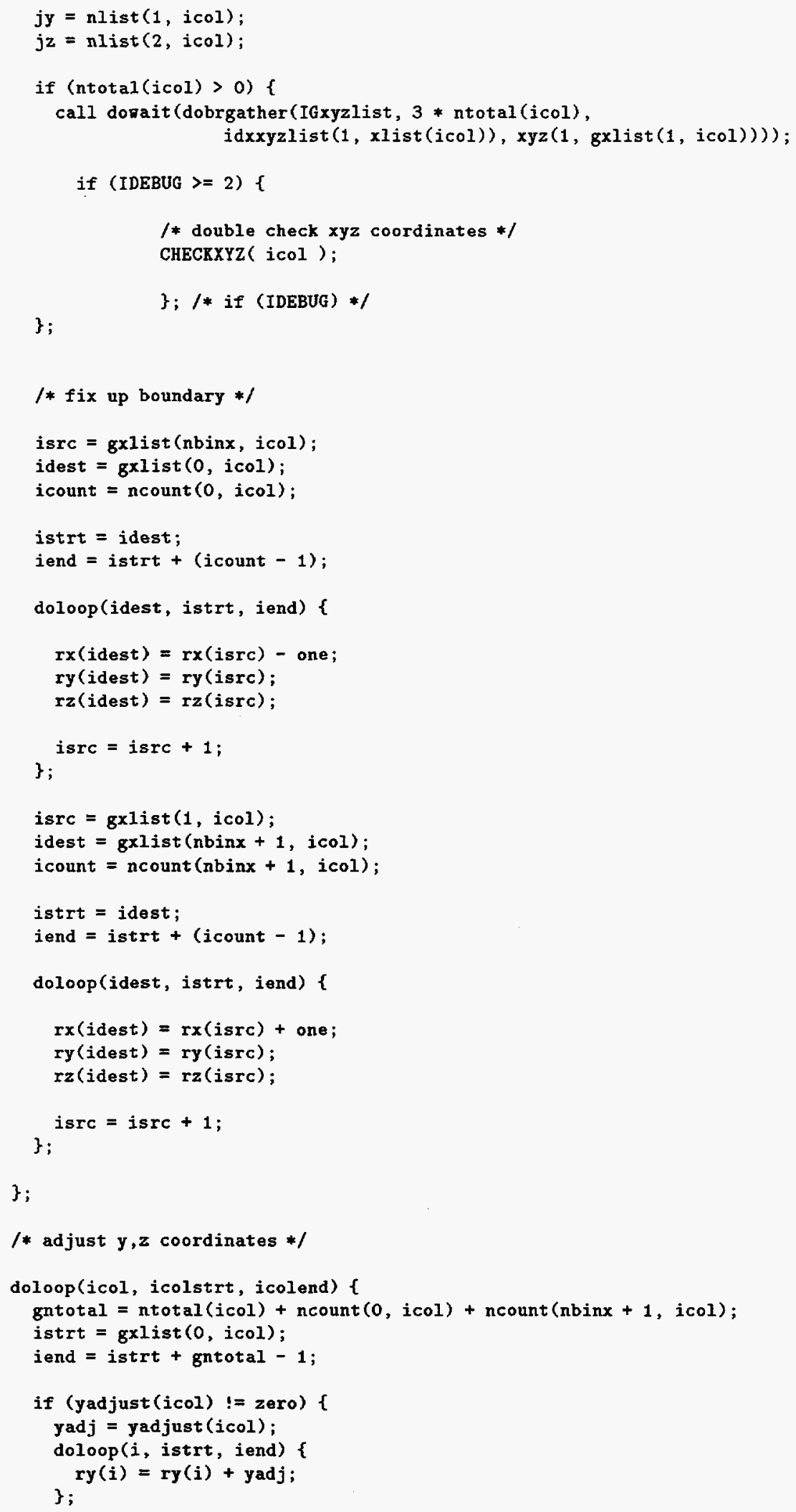




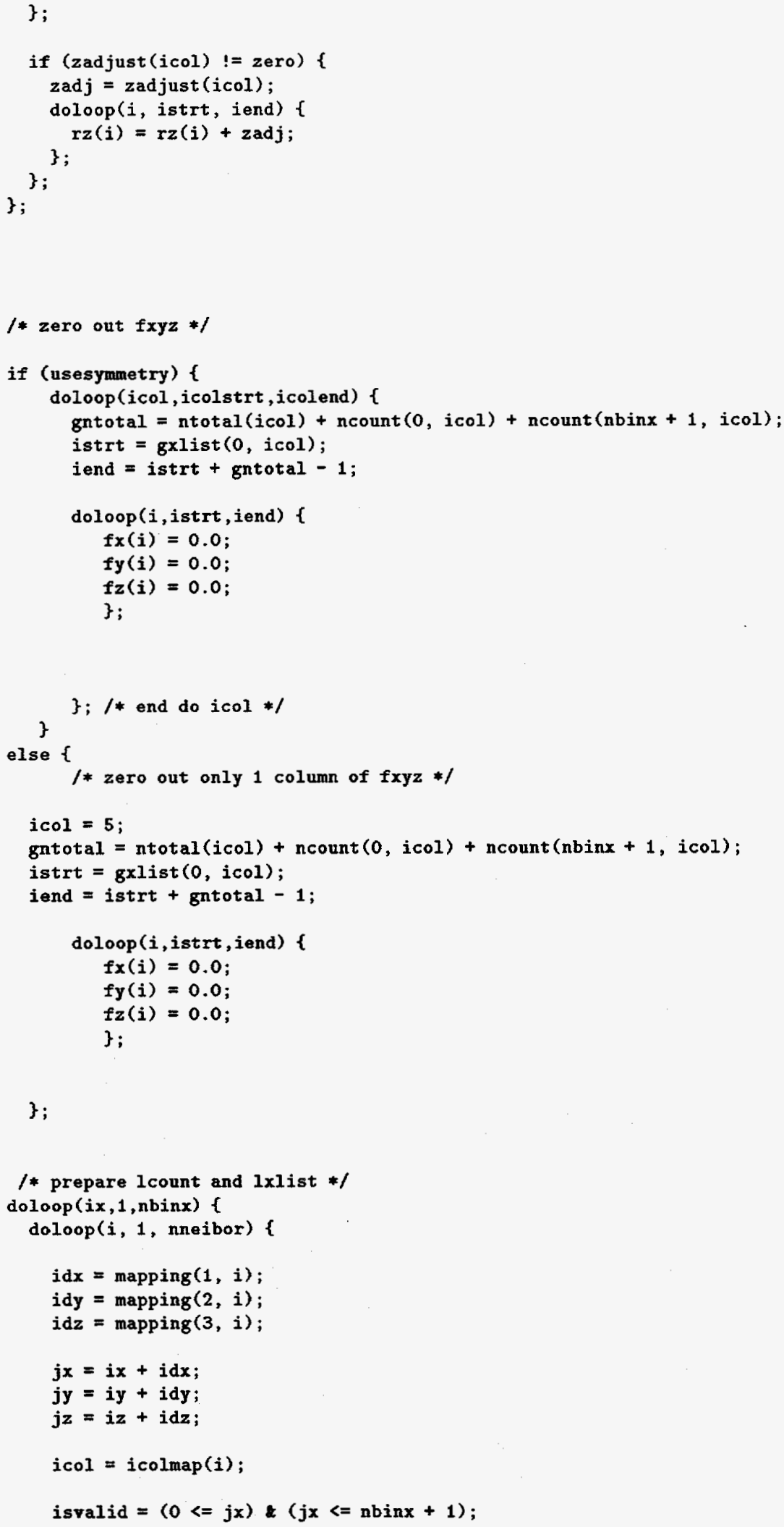




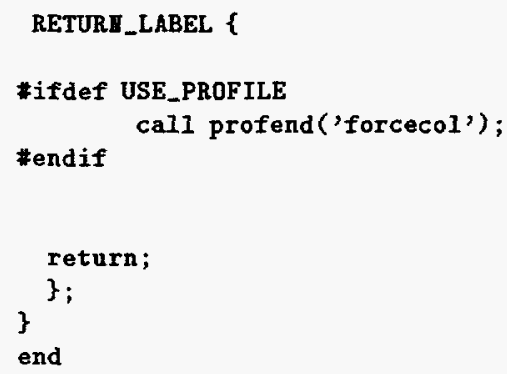

4.11. subroutine forcecal

Finclude "stdinc.h"

*undef ORIGIHAL

\#define USE_ABS

\#ifdef MP

*undef USE_PROFILE

\#endif

*define RETURI_LABEL. 9999

\#ifdef USE_SYHHETRY

\#ifndef nneibor

*define nneibor 14

\#endif

\section{*else}

\#ifndef nneibor

*define nneibor 27

*endif

Fendif $/ *$ USE_SYMMETRY */

*define maxneibor 27

subroutine forcecal ( $i x, i y, i z$, ncount, $1 \times 1$ ist, ndim, fxyz, $x y z$, vpos, vneg, wpos, ,nneg )

\#include "globals.h"

integer $i x, i y, i z$

integer ncount (nneibor), $1 \times 1$ ist (nneibor); integer ndim;

real $x y z(3$, ndim $)$; 


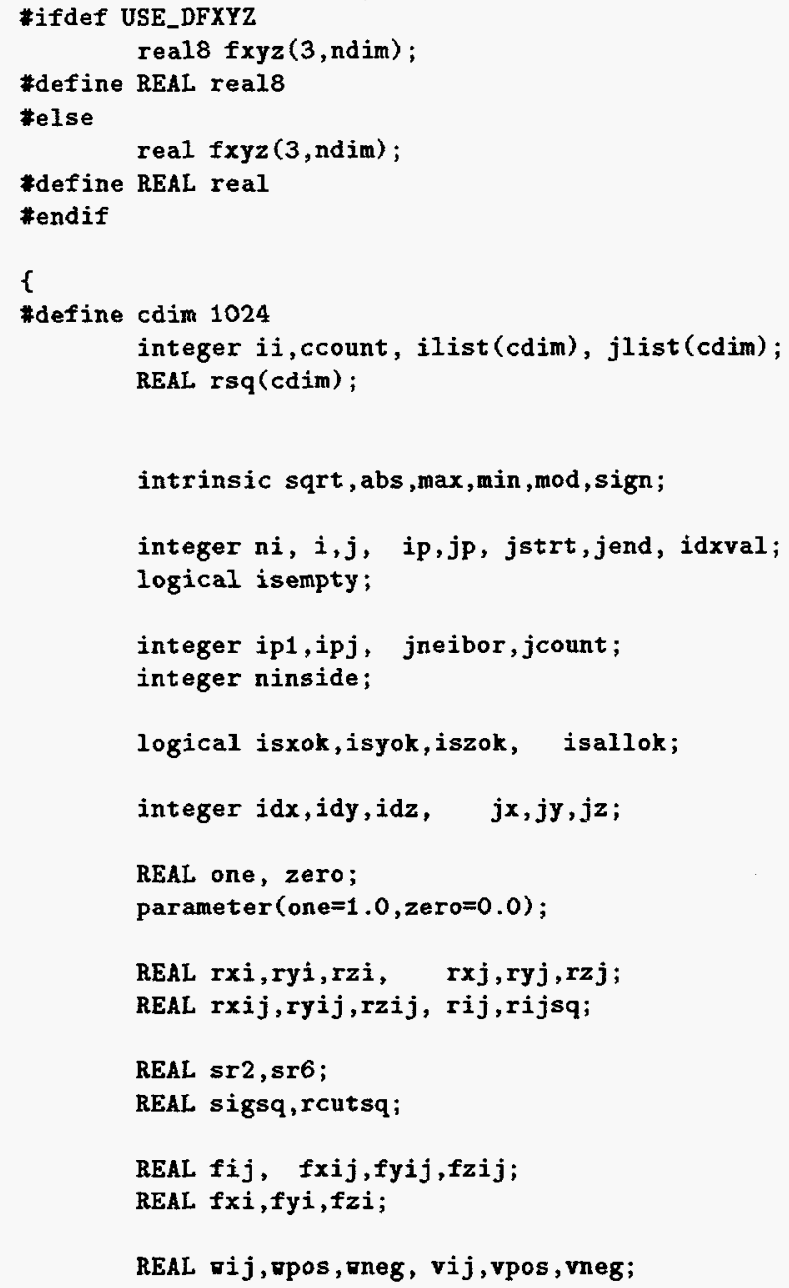

*dir\$r noconcur 


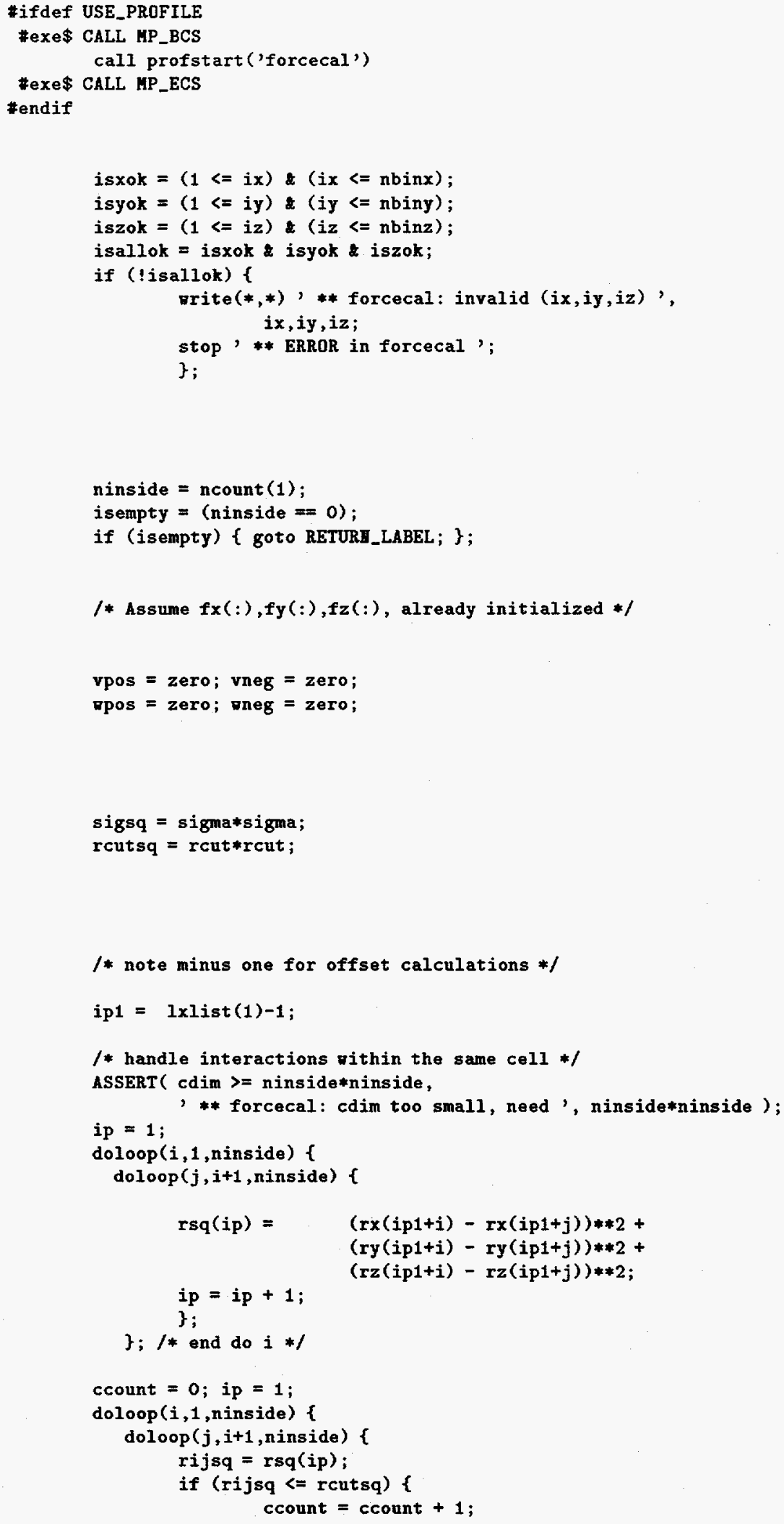




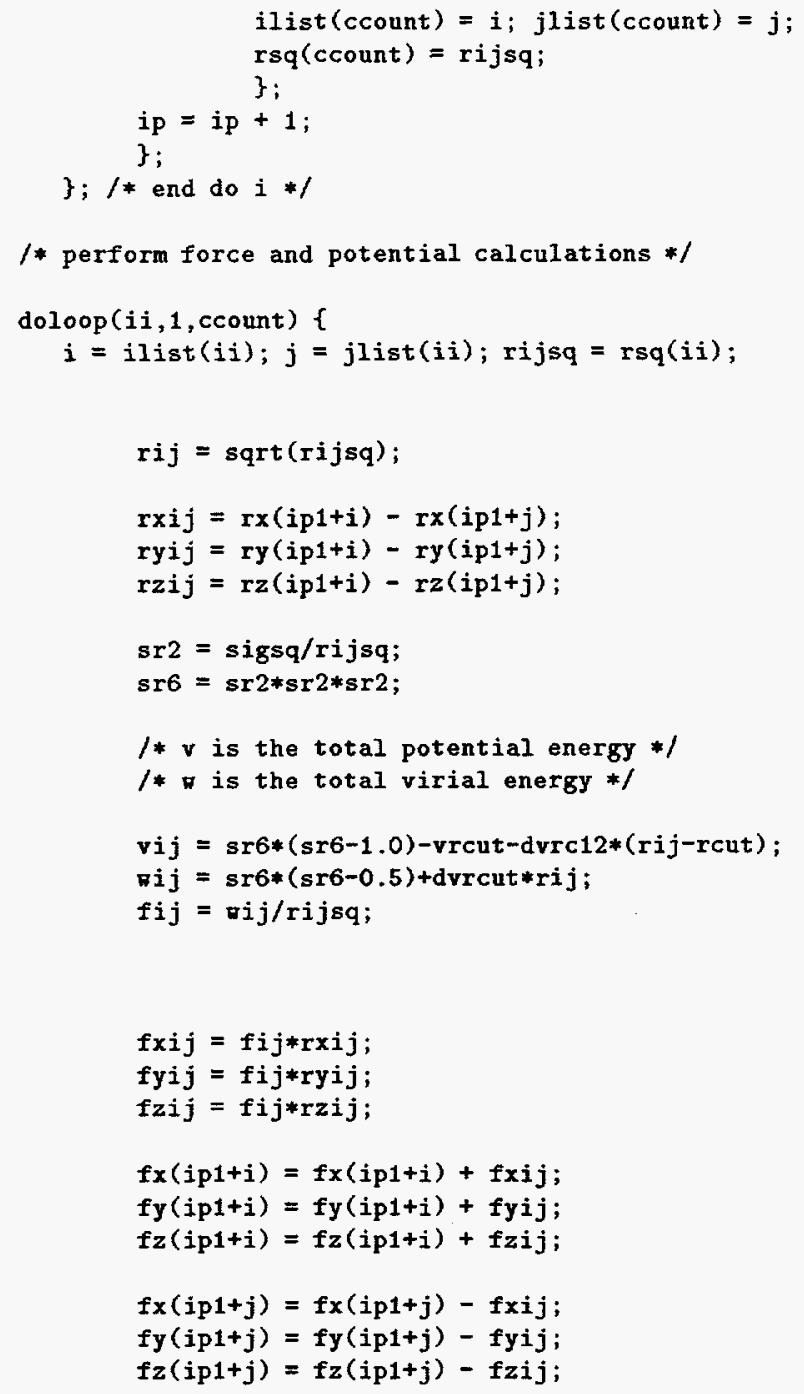




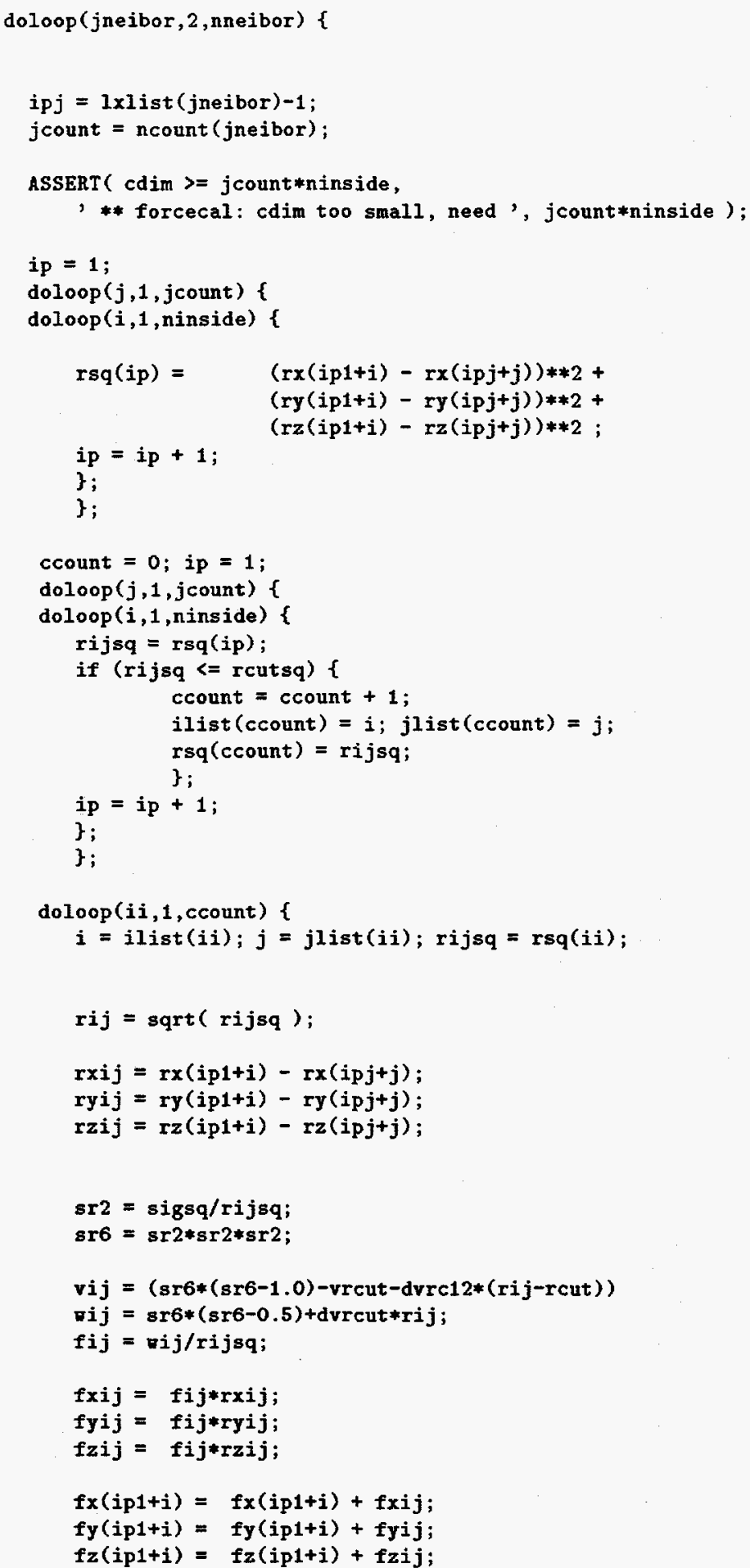

\#ifdef USE_SYMMETRY

$f x(i p j+j)=f x(i p j+j)-f x i j ;$

$f y(i p j+j)=f y(i p j+j)-f y i j$; 


$$
f z(i p j+j)=f z(i p j+j)-f z i j
$$

*else /* USE_SYHHETRY */

$$
\begin{aligned}
& v i j=0.5 * v i j \\
& \nabla i j=0.5 * \nabla i j
\end{aligned}
$$

*endif /* USE_SYMMETRY */

\#ifdef USE_ABS

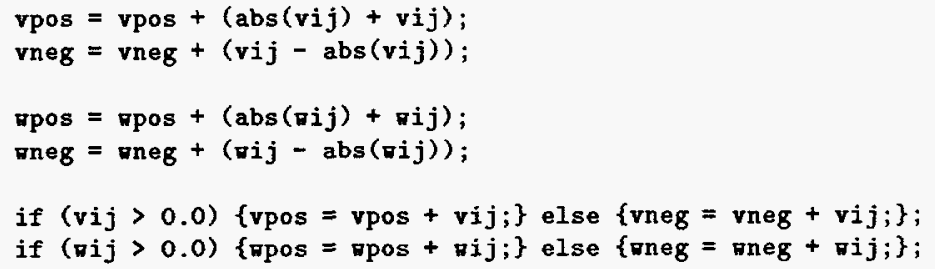

\#endif $/ *$ USE_ABS */

\}; $/ *$ end do ii *f

\}; /* end do jneigbor *I

\#ifdef USE_ABS

vpos $=0.5 *$ vpos; vneg $=0.5 *$ vneg;

upos $=0.5 *$ tpos; toneg $=0.5 *$ wneg;

\#endif $/ *$ USE_ABS */

/* summation into global $\mathrm{v}$ and $\mathrm{x}$ performed in forcecol */

RETURI_LABEI \{

\#ifdef USE_PROFILE

\#exe $\$$ CALL MP_BCS

call profend('forcecal')

\#exe\$ CALL MP_ECS

*endif

return;

\};

\}

end 


\section{References}

[1] M. P. Allen And D. Tildesley, Computer Simulation of Liquids, Claredon Press, Oxford, 1987.

[2] D. M. Beasley ANd P. S. Lomdahl, Message passing multi-cell molecular dynamics on the Connection Machine 5, Parall. Comp., 20 (1994), pp. 173196.

[3] E. F. D'Azevedo And C. H. Romine, DOLIB: Distributed Object Library, Tech. Report ORNL/TM-12744, Oak Ridge National Laboratory, 1994.

[4] R. W. Hockney, S. P. Goel, and J. W. Eastwood, Quiet highresolution computer models of a plasma, J. Comp. Phys, 14 (1974), pp. 148158.

[5] P. S. Lomdahl, P. Tamayo, N. Gronbech-Jensen, and D. M. BeaZLEY, 50 GFlops molecular dynamics on the Connection Machine 5, in Proceedings Supercomputing '93, November 15-19, Portland, Oregon, Assocation for Computing Machinery and The Institute of Electrical and Electronics Engineers, Inc., 1993, pp. 520-527.

[6] B. Marr, R. PeIerls, and J. Pasciak, IPX - Preemptive remote procedure execution for concurrent applications, tech. report, Brookhaven National Laboratory, 1994.

[7] M. Pinches, D. Tildesley, And W. Smith, Large scale molecular dynamics on parallel computers using the link-cell algorithm., Molecular Simulation, 6 (1991), pp. 51-87. see also ftp://ftp.dl.ac.uk/ccp5/SOTON_PAR/README.

[8] S. Plimpton, Fast parallel algorithms for short-range molecular dynamics, Tech. Report SAND91-1144, UC-705, Sandia National Laboratories, Printed May 1993. 
[9] J. G. Powles, W. A. B. Evans, And N. QuIRKE, Non-destructive molecular dynamics simulation of the chemical potential of a fluid, Mol. Phys, 46 (1982), pp. 1347-1370.

[10] L. VERLET, Computer experiments on classical fluids I. Thermodynamical properties of Lennard-Jones molecules, Phys. Rev., 159 (1967), pp. 98-103. 


\section{INTERNAL DISTRIBUTION}

1. B. R. Appleton

2. B. A. Carreras

3-7. E. F. D'Azevedo

8. T. S. Darland

9. J. J. Dongarra

10. J. B. Drake

11. T. H. Dunigan

12. W. R. Emanuel

13. G. A. Geist

14. K. L. Kliewer

15-19. M. R. Leuze

20. E. G. $\mathrm{Ng}$

21. C. E. Oliver

22. B. W. Peyton

23-27. S. A. Raby
28. B. A. Riley

29-33. C. H. Romine

34. W. A. Shelton

35-39. R. F. Sincovec

40. G. M. Stocks

41. M. R. Strayer

42. D. W. Walker

43. P. H. Worley

44. T. Zacharia

45. Central Research Library

46. ORNL Patent Office

47. K-25 Applied Technology Library

48. Y-12 Technical Library

49. Laboratory Records - RC

50-51. Laboratory Records Dept.

\section{EXTERNAL DISTRIBUTION}

52. Loyce M. Adams, Applied Mathematics, FS-20, University of Washington, Seattle, WA 98195

53. Christopher R. Anderson, Department of Mathematics, University of California, Los Angeles, CA 90024

54. Todd Arbogast, Department of Mathematical Sciences, Rice University, P. O. Box 1892, Houston, TX 77251

55. Donald M. Austin, 6196 EECS Building, University of Minnesota, 200 Union Street, S.E., Minneapolis, MN 55455

56. Robert G. Babb, Oregon Graduate Center, CSE Department, 19600 N.W. Walker Road, Beaverton, OR 97006

57. David H. Bailey, NASA Ames, Mail Stop 258-5, NASA Ames Research Center, Moffet Field, CA 94035

58. Jesse L. Barlow, Department of Computer Science and Engineering, 220 Pond Laboratory, The Pennsylvania State University, University Park, PA 16802-6106

59. Edward H. Barsis, Computer Science and Mathematics, P. O. Box 5800, Sandia National Laboratory, Albuquerque, NM 87185

60. Adam Beguelin, Carnegie Mellon University, School of Computer Science, 5000 Forbes Avenue, Pittsburgh, PA 15213-3890

61. Robert E. Benner, Parallel Processing Division 1413, Sandia National Laboratories, P. O. Box 5800, Albuquerque, NM 87185 
62. Marsha J. Berger, Courant Institute of Mathematical Sciences, 251 Mercer Street, New York, NY 10012

63. Philippe Berger, Institut National Polytechnique, ENSEEIHT, 2 rue Charles CamichelF, 31071 Toulouse Cedex, France

64. Ake Bjorck, Department of Mathematics, Linkoping University, S-581 83 Linkoping, Sweden

65. John H. Bolstad, L-16, Lawrence Livermore National Laboratory, P. O. Box 808, Livermore, CA 94550

66. Roger W. Brockett, Harvard University, Pierce Hall, 29 Oxford Street Cambridge, MA 02138

67. James C. Browne, Department of Computer Sciences, University of Texas, Austin, TX 78712

68. Bill L. Buzbee, Scientific Computing Division, National Center for Atmospheric Research, P. O. Box 3000, Boulder, CO 80307

69. Donald A. Calahan, Department of Electrical and Computer Engineering, University of Michigan, Ann Arbor, MI 48109

70. Thomas A. Callcott, Director, The Science Alliance Program, 53 Turner House, University of Tennessee, Knoxville, TN 37996

71. Tony Chan, Department of Mathematics, University of California, Los Angeles, 405 Hilgard Avenue, Los Angeles, CA 90024

72. Jagdish Chandra, Army Research Office, P. O. Box 12211, Research Triangle Park, NC 27709

73. Siddhartha Chatterjee, Dept. of Computer Science, CB 3175, Sitterson Hall, The University of North Carolina, Chapel Hill, NC 27599-3175

74. Melvyn Ciment, National Science Foundation, 1800 G Street N.W., Washington, DC 20550

75. Tom Coleman, Department of Computer Science, Cornell University, Ithaca, NY 14853

76. Alva Couch, Department of Computer Science, Tufts University, Medford, MA 02155

77. Paul Concus, Mathematics and Computing, Lawrence Berkeley Laboratory, Berkeley, CA 94720

78. Tom Crockett, ICASE, Mail Stop 132C, NASA Langley Research Center, Hampton, VA 23665-5225

79. Jane K. Cullum, IBM T. J. Watson Research Center, P. O. Box 218, Yorktown Heights, NY 10598

80. George Cybenko, Center for Supercomputing Research and Development, University of Illinois, 104 South Wright Street, Urbana, IL 61801-2932

81. Helen Davis, Computer Science Department, Stanford University, Stanford, CA 94305

82. Michel Dayde, Institut National Polytechnique, ENSEEIHT, 2 rue Charles CamichelF, 31071 Toulouse Cedex, France 
83. Craig Douglas, IBM T. J. Watson Research Center, P. O. Box 218, Yorktown Heights, NY 10598-0218

84. Donald J. Dudziak, Department of Nuclear Engineering, 110B Burlington Engineering Labs, North Carolina State University, Raleigh, NC 27695-7909

85. Iain S. Duff, Atlas Centre, Rutherford Appleton Laboratory, Chilton, Oxon OX11 0QX, England

86. Victor Eijkhout, University of Tennessee, 107 Ayres Hall, Department of Computer Science, Knoxville, TN 37996-1301

87. Stanley Eisenstat, Department of Computer Science, Yale University, P. O. Box 2158 Yale Station, New Haven, CT 06520

88. Howard C. Elman, Computer Science Department, University of Maryland, College Park, MD 20742

89. Richard E. Ewing, Director, Institute for Scientific Computations, Texas A\&M University, College Station, TX 77843-3404

90. Edward Felten, Department of Computer Science, University of Washington, Seattle, WA 98195

91. Charles Fineman, Ames Research Center, Mail Stop 269/3, Moffet Field, CA 94035

92. David Fisher, Department of Mathematics, Harvey Mudd College, Claremont, CA 91711

93. Jon Flower, Parasoft Corporation, 2500 E. Foothill Boulevard, Suite 205, Pasadena, CA 91107

94. Geoffrey C. Fox, NPAC, 111 College Place, Syracuse University, Syracuse, NY $13244-4100$

95. Chris Fraley, Statistical Sciences, Inc., 1700 Westlake Avenue N, Suite 500, Seattle, WA 98119

96. Joan M. Francioni, Computer Science Department, University of Southwestern Louisiana, Lafayette, LA 70504

97. Paul O. Frederickson, ACL, MS B287, Los Alamos National Laboratory, Los Alamos, NM 87545

98. Offir Frieder, George Mason University, Science and Technology Building, Computer Science Department, 4400 University Drive, Fairfax, Va 22030-4444

99. Robert E. Funderlic, Department of Computer Science, North Carolina State University, Raleigh, NC 27650

100. Dennis B. Gannon, Computer Science Department, Indiana University, Bloomington, IN 47401

101. C. William Gear, NEC Research Institute, 4 Independence Way, Princeton, NJ 08540

102. W. Morven Gentleman, Division of Electrical Engineering, National Research Council, Building M-50, Room 344, Montreal Road, Ottawa, Ontario, Canada K1A 0R8 
103. Alan George, Vice President, Academic and Provost, Needles Hall, University of Waterloo, Waterloo, Ontario, Canada N2L 3G1

104. James Glimm, SUNY-Stony Brook, Department of Applied Mathematics and Statistics, Stony Brook, NY 11794

105. Gene Golub, Computer Science Department, Stanford University, Stanford, CA 94305

106. Joseph F. Grcar, Division 8245, Sandia National Laboratories, Livermore, CA 94551-0969

107. William D. Gropp, Mathematics and Computer Science Division, Argonne National Laboratory, 9700 South Cass Avenue, Argonne, IL 60439

108. Eric Grosse, AT\&T Bell Labs 2T-504, Murray Hill, NJ 07974

109. Sanjay Gupta, ICASE, Mail Stop 132C, NASA Langley Research Center, Hampton, VA 23665-5225

110. John L. Gustafson, Ames Laboratory, 236 Wilhelm Hall, Iowa State University, Ames, IA 50011-3020

111. Christian Halloy, Assistant Director of JICS, 104 South College, Joint Institute for Computational Science, University of Tennessee, Knoxville, TN 37996-1301

112. Sven J. Hammarling, The Numerical Algorithms Group, Ltd., Wilkinson House, Jordan Hill Road, Oxford OX2 8DR, United Kingdom

113. Robert M. Haralick, Department of Electrical Engineering, Director, Intelligent Systems Lab, University of Washington, 402 Electrical Engineering Building, FT10, Seattle, WA 98195

114. Ann H. Hayes, Computing and Communications Division, Los Alamos National Laboratory, Los Alamos, NM 87545

115. Michael T. Heath, National Center for Supercomputing Applications, 4157 Beckman Institute University of Illinois, 405 North Mathews Avenue, Urbana, IL 61801-2300

116. Gerald W. Hedstrom, L-71, Lawrence Livermore National Laboratory, P. O. Box 808, Livermore, CA 94550

117. Don E. Heller, Ames Laboratory, 327 Wilhelm, Ames, IA 50011

118. John L. Hennessy, CIS 208, Stanford University, Stanford, CA 94305

119. N. J. Higham, Department of Mathematics, University of Manchester, Gtr Manchester, M13 9PL, England

120. Dan Hitchcock, Office of Scientific Computing, ER-7, Applied Mathematical Sciences, Office of Energy Research, U.S. Department of Energy, Washington, DC 20585

121. Charles J. Holland, Air Force Office of Scientific Research, Building 410, Bolling Air Force Base, Washington, DC 20332

122. Fred Howes, Office of Scientific Computing, ER-7, Applied Mathematical Sciences, Office of Energy Research, Department of Energy, Washington, DC 20585

123. Robert E. Huddleston, Computation Department, Lawrence Livermore National Laboratory, P. O. Box 808, Livermore, CA 94550 
124. Jenq-Neng Hwang, Department of Electrical Engineering, FT-10, University of Washington, Seattle, WA 98195

125. Ilse Ipsen, Department of Computer Science, Yale University, P. O. Box 2158 Yale Station, New Haven, CT 06520

126. Leah H. Jamieson, School of Electrical Engineering, Purdue University, West Lafayette, IN 47907

127. Gary Johnson, Office of Scientific Computing, ER-7, Applied Mathematical Sciences, Office of Energy Research, U.S. Department of Energy, Washington, DC 20585

128. Lennart Johnsson, Thinking Machines Corporation, 245 First Street, Cambridge, MA 02142-1214

129. Harry Jordan, Department of Electrical and Computer Engineering, University of Colorado, Boulder, CO 80309

130. Bo Kagstrom, Institute of Information Processing, University of Umea, 5-901 87 Umea, Sweden

131. Malvyn Kalos, Cornell Theory Center, Engineering and Theory Center Building, Cornell University, Ithaca, NY 14853-3901

132. Hans Kaper, Mathematics and Computer Science Division, Argonne National Laboratory, 9700 South Cass Avenue, Bldg. 221, Argonne, IL 60439

133. Alan H. Karp, HP Labs 3U-7, Hewlett-Packard Company, 1501 Page Mill Road, Palo Alto, CA 94304

134. Linda Kaufman, Bell Laboratories, 600 Mountain Avenue, Murray Hill, NJ 07974

135. Robert J. Kee, Applied Mathematics Division 8245, Sandia National Laboratories, Livermore, CA 94551-0969

136. Kenneth Kennedy, Department of Computer Science, Rice University, P.O. Box 1892, Houston, TX 77001

137. Tom Kitchens, Office of Scientific Computing, ER-7, Applied Mathematical Sciences, Office of Energy Research, U.S. Department of Energy, Washington, DC 20585

138. Clyde P. Kruskal, Department of Computer Science, University of Maryland, College Park, MD 20742

139. Edward Kushner, Intel Corporation, 15201 NW Greenbrier Parkway, Beaverton, OR 97006

140. Michael Langston, Department of Computer Science, University of Tennessee, Knoxville, TN 37996-1301

141. Richard Lau, Office of Naval Research, Code 111MA 800 Quincy Street, Boston Tower 1, Arlington, VA 22217-5000

142. Robert L. Launer, Army Research Office, P. O. Box 12211, Research Triangle Park, NC 27709

143. Tom Leighton, Lab for Computer Science, Massachusetts Institute of Technology, 545 Technology Square, Cambridge, MA 02139 
144. James E. Leiss, Rt. 2, Box 142C, Broadway, VA 22815

145. Robert Leland, Sandia National Laboratories, 1424, P. O. Box 5800, Albuquerque, NM 87185-5800

146. Randall J. LeVeque, Applied Mathematics, FS-20, University of Washington, Seattle, WA 98195

147. John G. Lewis, Boeing Computer Services, P. O. Box 24346, M/S 7L-21, Seattle, WA 98124-0346

148. Heather M. Liddell, Center for Parallel Computing, Department of Computer Science and Statistics, Queen Mary College, University of London, Mile End Road, London E1 4NS, England

149. Brent Lindquist, SUNY-Stony Brook, Department of Applied Mathematics and Statistics, Stony Brook, NY 11794

150. Rik Littlefield, Pacific Northwest Laboratory, MS K1-87, P.O.Box 999, Richland, WA 99352

151. Joseph Liu, Department of Computer Science, York University, 4700 Keele Street, Downsview, Ontario, Canada M3J 1P3

152. Franklin Luk, Department of Computer Science, Amos Eaton Building - No. 131 Rensselaer Polytechnic Institute Troy, NY 12180-3590

153. Ewing Lusk, Mathematics and Computer Science Division, Argonne National Laboratory, 9700 South Cass Avenue, MCS 221 Argonne, IL 60439-4844

154. Allen D. Malony, Department of Computer and Information Science, University of Oregon, Eugene, OR 97403

155. Thomas A. Manteuffel, Department of Mathematics, University of Colorado Denver, Denver, CO 80202

156. Anita Mayo, IBM T. J. Watson Research Center, P. O. Box 218, Yorktown Heights, NY 10598

157. Oliver McBryan, University of Colorado at Boulder, Department of Computer Science, Campus Box 425, Boulder, CO 80309-0425

158. James McGraw, Lawrence Livermore National Laboratory, L-306, P. O. Box 808, Livermore, CA 94550

159. Piyush Mehrotra, ICASE, Mail Stop 132C, NASA Langley Research Center, Hampton, VA 23665

160. Paul C. Messina, Mail Code 158-79, California Institute of Technology, 1201 E. California Boulevard, Pasadena, CA 91125

161. Cleve B. Moler, MathWorks, 325 Linfield Place, Menlo Park, CA 94025

162. Neville Moray, Department of Mechanical and Industrial Engineering, University of Illinois, 1206 West Green Street, Urbana, IL 61801

163. Jorge J. More, Mathematics and Computer Science Division, Argonne National Laboratory, 9700 South Cass Avenue, Argonne, IL 60439

164. William A. Mulder, Koninklijke Shell Exploratie en Produktie Laboratorium, Postbus 60, 2280 AB Rijswijk, The Netherlands 
165. David Nelson, Director, Office of Scientific Computing, ER-7, Applied Mathematical Sciences, Office of Energy Research, U.S. Department of Energy, Washington, DC 20585

166. V. E. Oberacker, Department of Physics, Vanderbilt University, Box 1807, Station B, Nashville, TN 37235

167. Dianne P. O'Leary, Computer Science Department, University of Maryland, College Park, MD 20742

168. Joseph Oliger, Computer Science Department, Stanford University, Stanford, CA 94305

169. James M. Ortega, Department of Computer Science, Thornton Hall, University of Virginia, Charlottesville, VA 22901

170. Steve Otto, Oregon Graduate Institute, Department of Computer Science and Engineering, $19600 \mathrm{NW}$ von Neumann Drive, Beaverton, OR 97006-1999

171. Cherri Pancake, Department of Computer Science, Oregon State University, Corvallis, OR 97331-3202

172. Joseph E. Pasciak, Applied Mathematics, Brookhaven National Laboratory, Upton, NY 11973

173. Merrell Patrick, National Science Foundation, 1800 G Street N.W., Washington, DC 20550

174. David Payne, Intel Corporation, Supercomputer Systems Division, $15201 \mathrm{NW}$ Greenbrier Parkway, Beaverton, OR 97006

175. Ronald F. Peierls, DAS - Bldg. 490-D, P.O. Box 5000, Brookhaven National Laboratory, Upton, NY 11973

176. Linda R. Petzold, Computer Science Department, University of Minnesota, 200 Union Street, S.E., Room 4-192, Minneapolis, MN 55455

177. Dan Pierce, Boeing Computer Services, P.O. Box 24346, M/S 7L-21, Seattle, WA 98124-0346

178. Paul Pierce, Intel Corporation, Supercomputer Systems Division, 15201 NW Greenbrier Parkway, Beaverton, OR 97006

179. Robert J. Plemmons, Departments of Mathematics and Computer Science, North Carolina State University, Raleigh, NC 27650

180. James C. T. Pool, Deputy Director, Caltech Concurrent Supercomputing Facility, California Institute of Technology, MS 158-79, Pasadena, CA 91125

181. Jesse Poore, Computer Science Department, University of Tennessee, Knoxville, TN 37996-1300

182. David A. Poplawski, Department of Computer Science, Michigan Technological University, Houghton, MI 49931

183. Roldan Pozo, University of Tennessee, 107 Ayres Hall, Department of Computer Science, Knoxville, TN 37996-1301

184. Padma Raghavan, University of Illinois, NCSA, 4151 Beckman Institute, 405 North Matthews Avenue, Urbana, IL 61801 
185. Daniel A. Reed, Computer Science Department, University of Illinois, Urbana, IL 61801

186. John K. Reid, Numerical Analysis Group, Central Computing Department, Atlas Centre, Rutherford Appleton Laboratory, Didcot, Oxon OX11 0QX, England

187. John R. Rice, Computer Science Department, Purdue University, West Lafayette, IN 47907

188. Garry Rodrigue, Numerical Mathematics Group, Lawrence Livermore National Laboratory, Livermore, CA 94550

189. Donald J. Rose, Department of Computer Science, Duke University, Durham, NC 27706

190. Ahmed H. Sameh, Department of Computer Science, University of Minnesota, 200 Union Street S.E., Minneapolis, MN 55455

191. Joel Saltz, Computer Science Department, A.V. Williams Building, University of Maryland, College Park, MD 20742

192. Jorge Sanz, IBM Almaden Research Center, Department K53/802, 650 Harry Road, San Jose, CA 95120

193. Robert B. Schnabel, Department of Computer Science, University of Colorado at Boulder, ECOT 7-7 Engineering Center, Campus Box 430, Boulder, CO 803090430

194. Robert Schreiber, RIACS, MS 230-5, NASA Ames Research Center, Moffet Field, CA 94035

195. Martin H. Schultz, Department of Computer Science, Yale University, P. O. Box 2158 Yale Station, New Haven, CT 06520

196. David S. Scott, Intel Scientific Computers, 15201 N.W. Greenbrier Parkway, Beaverton, OR 97006

197. The Secretary, Department of Computer Science and Statistics, The University of Rhode Island, Kingston, RI 02881

198. Charles L. Seitz, Department of Computer Science, California Institute of Technology, Pasadena, CA 91125

199. Margaret L. Simmons, Computing and Communications Division, Los Alamos National Laboratory, Los Alamos, NM 87545

200. Horst D. Simon, NASA Ames Research Center, Mail Stop T045-1, Moffett Field, CA 94035

201. William C. Skamarock, 3973 Escuela Court, Boulder, CO 80301

202. Tony Skjellum, Dept of Computer Science, Mississippi State University, PO Drawer CS, Mississippi State, MS 39762-5623

203. Burton Smith, Tera Computer Company, 400 North 34th Street, Suite 300, Seattle, WA 98103

204. Marc Snir, IBM T.J. Watson Research Center, Department 420/36-241, P. O. Box 218, Yorktown Heights, NY 10598

205. Larry Snyder, Department of Computer Science and Engineering, FR-35, University of Washington, Seattle, WA 98195 
206. Danny C. Sorensen, Department of Mathematical Sciences, Rice University, P. O. Box 1892, Houston, TX 77251

207. Rick Stevens, Mathematics and Computer Science Division, Argonne National Laboratory, 9700 South Cass Avenue, Argonne, IL 60439

208. G. W. Stewart, Computer Science Department, University of Maryland, College Park, MD 20742

209. Paul N. Swarztrauber, National Center for Atmospheric Research, P. O. Box 3000, Boulder, CO 80307

210. Julie Swisshelm, Sandia National Laboratories, 1421, Parallel Computational Sciences Department, Albuquerque, New Mexico 87185-5800

211. Wei Pai Tang, Department of Computer Science, University of Waterloo, Waterloo, Ontario, Canada N2l 3G1

212. Bernard Tourancheau, LIP ENS-Lyon 69364, Lyon cedex 07, France

213. Joseph F. Traub, Department of Computer Science, Columbia University, New York, NY 10027

214. Lloyd N. Trefethen, Department of Computer Science, Cornell University, Ithaca, NY 14853

215. Robert van de Geijn, University of Texas, Department of Computer Sciences, TAI 2.124, Austin, TX 78712

216. Charles Van Loan, Department of Computer Science, Cornell University, Ithaca, NY 14853

217. Udaya B. Vemulapati, Department of Computer Science, University of Central Florida, Orlando, FL 32816-0362

218. Robert G. Voigt, National Science Foundation, Room 417, 1800 G Street N.W., Washington, DC 20550

219. Bi R. Vona, Center for Numerical Analysis, RLM 13.150, University of Texas at Austin, Austin, TX 78712

220. Henk A. van der Vorst, Professor Dept. of Mathematics, Universiteit Utrecht, P.O. Box 80010, 3508 TA, Utrecht, THE NETHERLANDS

221. Michael D. Vose, 107 Ayres Hall, Department of Computer Science, University of Tennessee, Knoxville, TN 37996-1301

222. Phuong Vu, Cray Research, Inc., 19607 Franz Road, Houston, TX 77084

223. A. J. Wathen, School of Mathematics, University Walk, Bristol BSB 1TW, England

224. Robert P. Weaver, 1555 Rockmont Circle, Boulder, CO 80303

225. Mary F. Wheeler, Department of Mathematical Sciences, Rice University, P. O. Box 1892, Houston, TX 77251

226. Andrew B. White, Computing Division, Los Alamos National Laboratory, Los Alamos, NM 87545

227. John Zahorjan, Department of Computer Science and Engineering, Sieg Hall, FR35, University of Washington, Seattle, WA 98195 
228. Office of Assistant Manager for Energy Research and Development, U.S. Department of Energy, Oak Ridge Operations Office, P. O. Box 2001, Oak Ridge, TN $37831-8600$

229-230. Office of Scientific \& Technical Information, P. O. Box 62, Oak Ridge, TN 37831 